\title{
Heterogeneous beliefs and asset pricing in discrete time: an analysis of pessimism and doubt
}

\author{
Elyès JOUINI* Clotilde NAPP ${ }^{\dagger}$
}

July 7,2006

\begin{abstract}
The aim of the paper is to analyze the impact of heterogeneous beliefs in an otherwise standard competitive complete markets discrete time economy. The construction of a consensus belief, as well as a consensus consumer are shown to be valid modulo a predictable aggregation bias, which takes the form of a discount factor. We use our construction of a consensus consumer to investigate the impact of beliefs heterogeneity on the CCAPM and on the expression of the risk free rate. We focus on the pessimism/doubt of the consensus consumer and we study their impact on the equilibrium characteristics (market price of risk, risk free rate). We finally analyze how pessimism and doubt at the aggregate level result from pessimism and doubt at the individual level.
\end{abstract}

\section{Introduction}

The aim of the paper is to analyze the impact of heterogeneous beliefs in an otherwise standard competitive complete markets discrete time economy.

${ }^{*}$ Corresponding author. Institut universitaire de France and CEREMADE, Université de Paris IX Dauphine, Place du Maréchal de Lattre de Tassigny, 75116 Paris. e-mail : jouini@ceremade.dauphine.fr

${ }^{\dagger}$ CEREMADE, Université de Paris IX Dauphine, Place du Maréchal de Lattre de Tassigny, 75116 Paris and CREST. e-mail : napp@ceremade.dauphine.fr. 
We start from a given equilibrium with heterogeneous beliefs in a fairly general setting and we propose to decompose our analysis into three steps.

The first issue deals with the aggregation of these heterogeneous beliefs. The main question is to know if it is possible to analyze the heterogeneous beliefs model in terms of a classical homogeneous beliefs model, in which the common belief may be different from the objective one. In other words, can we define a subjective consensus belief, i.e. a belief which, if held by all individuals, would generate the same equilibrium prices as in the actual heterogeneous economy?

We show that the answer to the previous question is positive. We are then led to analyze, in a standard homogeneous model, the impact of a subjective belief on the equilibrium characteristics (state price density, risk premium, risk free rate, etc.). This question has been explored by Abel (2002) in the specific setting of power utility functions and i.id asset returns. In particular, Abel introduces concepts of pessimism and doubt, and analyzes their impact on the equilibrium characteristics. The concepts introduced by Abel (2002) are not suited for our framework and we introduce alternative concepts that have an unambiguous impact on the equilibrium characteristics in a more general setting (in particular, without specific restrictions on the utility functions nor on the dynamics and distribution of the asset returns).

The last question is to understand how doubt and pessimism at the individual level are captured at the aggregate level, i.e. by the consensus belief. Our definitions of doubt and pessimism appear as particularly adapted to this question. Indeed, roughly speaking, we obtain that the level of pessimism (resp. doubt) at the aggregate level is a weighted average of the level of pessimism (resp. doubt) at the individual level.

In this paper, the different subjective beliefs are considered as given. As in Varian (1985, 1989), Abel (1989) or Harris-Raviv (1993), they reflect difference of opinion among the agents rather than difference of information; indeed, "we assume that investors receive common information, but differ in the way they interpret this information" (Harris-Raviv, 1993). The different subjective beliefs might come from a Bayesian updating of the investors predictive distribution over the uncertain returns on risky securities as in, e.g. Williams (1977), DetempleMurthy (1994), Zapatero (1998), Gallmeyer (2000), Basak (2000), Gallmeyer and Hollifield (2002), but we do not make such an assumption; we only impose that the subjective probabilities be equivalent to the initial one. Notice that the abovementioned models with learning are not "more endogenous", since the investors' updating rule and the corresponding probabilities can be determined separately 
from his/her optimization problem (see e.g. Genotte, 1986).

In a companion paper, Jouini and Napp (2004) considered the same problem in continuous time. Even if the approach developed therein is similar to the one developed here, the techniques, the concepts and the results are quite different and the contribution of this paper goes much further than a technical adaptation of the continuous time results. In particular, if we analyze the subjective beliefs in terms of distortions of the objective one, the discrete time setting permits us a much larger set of possible distortions than the continuous time setting. For instance, in continuous time, the volatility of a given process is the same under the objective and the different subjective beliefs (by Girsanov's Theorem) whereas in discrete time some agents might overestimate or underestimate this volatility leading to doubtful or overconfident behavior. More generally, in continuous time (again by Girsanov's Theorem) agents can only disagree on the instantaneous rates of returns of the assets. Therefore, there is only one degree of possible divergence between agents and each individual belief is measured by a one-dimensional parameter. The situation is much more complicated in discrete time since there are many ways of constructing a probability, which is equivalent to a given initial one, which leads to a much richer typology of possible beliefs.

The main contributions of this paper are:

- To construct a representative agent in the heterogeneous beliefs framework such that utility functions are aggregated into a representative utility function, beliefs are aggregated into a consensus belief and time discount factors are aggregated into a time discount factor. The representative utility function is an average of the individual ones and constructed as in the standard case, the consensus belief is an average of the individual ones and there is only a bias due to beliefs heterogeneity in the discount factor.

- To provide in a very general setting a simple way to analyze the impact of beliefs heterogeneity on equilibrium characteristics. Through our construction, the heterogeneous beliefs framework can be analyzed in terms of subjective beliefs in a homogeneous beliefs framework.

- To introduce concepts of pessimism and doubt at the individual level that have good aggregation properties and that have a clear impact on the equilibrium risk premium and risk free rate in a fairly general setting.

- To decompose into three distinct effects the impact of beliefs aggregation into a consensus belief, namely an average effect, a cautiousness effect and a relative weights effect and to show that a potential important source of pessimism/doubt at the aggregate level is the correlation between individual risk tolerance and the 
individual level of pessimism/doubt. In light of the risk premium and risk free rate puzzles (Mehra-Prescott, 1985, Weil, 1989), we show that if agents are on average pessimistic, there is a bias towards a higher market price of risk and a lower risk free rate than in the standard setting. Furthermore, if risk tolerance and pessimism are positively correlated, this induces a higher market price of risk.

The paper is organized as follows. We present in Section 2 the aggregation procedure that transforms the study of a model under heterogeneous subjective beliefs into the study of a model under homogeneous subjective beliefs and permits us to derive an adjusted CCAPM formula.

The next step consists in the study of the impact of a subjective belief on the equilibrium risk premium and risk free rate, which is the aim of Section 3. More precisely, we try to determine characteristics of the subjective belief that have an unambiguous impact on the market price of risk and on the risk free rate, and, in relation with the risk premium puzzle (see Mehra-Prescott, 1985 or Kocherlakota, 1996 for a survey on the risk premium puzzle) and the risk free rate puzzle (Weil, 1989), which preferably lead to an increase of the market price of risk and a decrease of the risk free rate.

The aim of Section 4 is to analyze how pessimism and doubt at the aggregate level result from pessimism and doubt at the individual level.

All proofs are in the appendix.

\section{Consensus belief, adjusted CCAPM and risk free rate}

In the classical representative agent approach, all investors are assumed to know the true probability distribution and in this section, we analyze to which extent this approach can be extended to heterogeneous subjective beliefs. More precisely, we start from a given equilibrium with heterogeneous beliefs in an otherwise standard complete discrete time market model, and we explore to which extent it is possible 1) to define a consensus belief, i.e. a belief, which, if held by all individuals would generate the same equilibrium prices as in the actual heterogeneous economy and 2) to define a representative agent (or a consensus consumer). We shall then analyze the impact of heterogeneity of beliefs on asset pricing, and more precisely on the CCAPM formula and on the expression of the risk free rate.

The model is standard, except that we allow the agents to have distinct subjective probabilities. We fix a finite time horizon $T$ on which we are going to treat our problem. We consider a filtered probability space $\left(\Omega,\left(F_{t}\right)_{t \in\{0, \ldots, T\}}, P\right)$, 
where the filtration $\left(F_{t}\right)_{t \in\{0, \ldots, T\}}$ satisfies the usual conditions. Each investor indexed by $i=1, \cdots, N$ solves a standard dynamic utility maximization problem. He has a current income at date $t$ denoted by $e_{t}^{*^{i}}$ and a von Neumann-Morgenstern utility function for consumption of the form $E^{Q^{i}}\left[\sum_{t=0}^{T} u_{i}\left(t, c_{t}\right)\right]$, where $Q^{i}$ is a probability measure equivalent to $P$ which corresponds to the subjective belief of individual $i$. If we denote by $\left(M_{t}^{i}\right)_{t \in\{0, \ldots, T\}}$ the positive density process of $Q^{i}$ with respect to $P$, then the utility function can be rewritten as $E^{P}\left[\sum_{t=0}^{T} M_{t}^{i} u_{i}\left(t, c_{t}\right)\right]$.

The different subjective beliefs might result from differences of opinions or from incomplete information and Bayesian updating. The origin of beliefs heterogeneity does not impact our analysis. In order to take into account Bayesian updating models, it suffices in such models to determine the individual beliefs (since, as underlined by Genotte (1986), the inverstor's updating rules and the corresponding probabilities can be determined separately from his/her optimization problem) and to conduct our analysis.

We make the following classical assumptions on the utility functions and on the subjective beliefs.

\section{Assumption}

- for all $t=1, \ldots, T, u_{i}(t, \cdot):\left[k_{i}, \infty\right) \rightarrow \mathbb{R} \cup\{-\infty\}$ is of class $C^{1}$ on $\left(k_{i}, \infty\right)$, strictly increasing and strictly concave ${ }^{1}$,

- $u_{i}(., c)$ and $u_{i}^{\prime}(t,)=.\frac{\partial u_{i}}{\partial c}(t,$.$) are continuous on [0, T]$,

- for $i=1, \cdots, N, P\left\{e_{t}^{i}>k_{i}\right\}>0$ and $P\left\{e_{t}^{i} \geq k_{i}\right\}=1$,

- there exists $\varepsilon>0$ such that $e^{*} \equiv \sum e^{i}>\sum_{i=1}^{N} k_{i}+\varepsilon, P$ a.s.,

- $E^{P}\left[\sum_{t=0}^{T} e_{t}^{*}\right]<\infty$

- the density process $M^{i}$ is uniformly bounded for $i=1, \cdots, N$.

The third condition can be seen as a survival assumption at the individual level and the fourth one as a survival assumption at the aggregate level. All the remaining conditions are very classical ones.

We do not specify the utility functions $u_{i}$, although we shall focus on the classical cases of linear risk tolerance utility functions (which include logarithmic, power as well as exponential utility functions).

\footnotetext{
${ }^{1}$ Note that we could easily generalize the results of this paper to the case where $k_{i}$ is a function of $t$.
} 


\subsection{Consensus belief}

We start from an equilibrium $\left(q^{*},\left(y^{*^{i}}\right)\right)$ relative to the beliefs $\left(M^{i}\right)$ and the income processes $e^{i}$. We recall that an equilibrium relative to the beliefs $\left(M^{i}\right)$ and the income processes $\left(e^{i}\right)$ is defined by a positive, uniformly bounded price process $q^{*}$ and a family of optimal admissible consumption plans $\left(y^{*^{*}}\right)$ such that markets clear i.e. a family of adapted $\left[k_{i}, \infty\right)$-valued processes $y^{*^{i}}$ such that $E^{P}\left[\sum_{0}^{T}\left|y_{t}^{*^{*}}\right|\right]<\infty$ and satisfying

$$
\left\{\begin{array}{c}
y^{*^{i}}=y^{i}\left(q^{*}, M^{i}, e^{i}\right) \\
\sum_{i=1}^{N} y^{*^{i}}=\sum_{i=1}^{N} e^{i} \equiv e^{*}
\end{array}\right.
$$

where

$$
y^{i}(q, M, e) \equiv \arg \max _{E^{P}\left[\sum_{0}^{T} q_{t}\left(y_{t}^{i}-e_{t}\right)\right] \leq 0} E^{P}\left[\sum_{0}^{T} M_{t} u_{i}\left(t, c_{t}\right) d t\right] .
$$

Such an equilibrium, when it exists, can be characterized by the first order necessary conditions for individual optimality and the market clearing condition. These conditions can be written as follows

$$
\left\{\begin{array}{c}
M_{t}^{i} u_{i}^{\prime}\left(t, y_{t}^{*^{i}}\right) \leq \lambda_{i} q_{t}^{*}, \quad \text { on }\left\{y^{*^{i}}=k_{i}\right\} \\
M_{t}^{i} u_{i}^{\prime}\left(t, y_{t}^{*^{*}}\right)=\lambda_{i} q_{t}^{*}, \quad \text { on }\left\{y^{*^{i}}>k_{i}\right\} \\
E^{P}\left[\sum_{0}^{T} q_{t}^{*}\left(y_{t}^{*^{i}}-e_{t}^{i}\right)\right]=0 \\
\sum_{i=1}^{N} y^{*^{i}}=e^{*}
\end{array}\right.
$$

for some set of positive Lagrange multipliers $\left(\lambda_{i}\right)$.

In the next, we will say that $\left(q^{*},\left(y^{*^{*}}\right)\right)$ is an interior equilibrium relative to the beliefs $\left(M^{i}\right)$ and the income processes $\left(e^{i}\right)$ if $y^{*^{i}}>k_{i}, P$ a.s. for $i=1, \cdots, N^{2}$.

Our first aim is to find an "equivalent equilibrium" in which the heterogeneous subjective beliefs would be aggregated into a common characteristic $M$. We shall define an "equivalent equilibrium" by two requirements. First, the "equivalent

${ }^{2}$ Note that under the following additional condition

$$
u_{i}^{\prime}\left(t, k_{i}\right)=\infty \text { for } t \in\{0, \ldots, T\} \text { and } i=1, \cdots, N,
$$

all the equilibria are interior ones. 
equilibrium" should generate the same equilibrium trading volumes $\left(y^{*^{i}}-e^{i}\right)$ and price process $q^{*}$ as in the original equilibrium with heterogeneous beliefs. Second, every investor should be indifferent at the margin between investing one additional unit of income in the original equilibrium with heterogeneous beliefs and in the "equivalent equilibrium", so that each asset gets the same marginal valuation by each investor (in terms of his marginal utility) in both equilibria ${ }^{3}$. We give credit to Calvet et al. (2004) for the idea of constructing an equivalent equilibrium satisfying the two mentioned requirements. However, Calvet et al. (2004) allow an adjustment of the aggregate wealth while, in our construction, we keep the aggregate wealth unchanged.

The existence of such an "equivalent equilibrium" is given by the following proposition.

Proposition 2.1. Consider an interior equilibrium $\left(q^{*},\left(y^{*^{i}}\right)\right)$ relative to the beliefs $\left(M^{i}\right)$ and the income processes $\left(e^{i}\right)$. There exist a unique positive and adapted process $\left(M_{t}\right)_{t \in\{0, \ldots, T\}}$ with $M_{0}=1$, a unique family of income processes $\left(\bar{e}^{i}\right)$ with $\sum_{i=1}^{N} \bar{e}^{i}=e^{*}$ and a unique family of individual consumption processes $\left(\bar{y}^{i}\right)$ such that $\left(q^{*},\left(\bar{y}^{i}\right)\right)$ is an equilibrium relative to the common characteristic $M$ and the income processes $\left(\bar{e}^{i}\right)$ and such that trading volumes and individual marginal valuation remain the same, i.e.

$$
\begin{aligned}
y^{*^{i}}-e^{i} & =\bar{y}^{i}-\bar{e}^{i} \quad i=1, \cdots, N \\
M_{t}^{i} u_{i}^{\prime}\left(t, y^{*^{i}}\right) & =M_{t} u_{i}^{\prime}\left(t, \bar{y}^{i}\right), \quad t \in\{0, \ldots, T\}, i=1, \cdots, N .
\end{aligned}
$$

This means that $\left(q^{*},\left(\bar{y}^{i}\right)\right)$ is an equilibrium with income transfers relative to the common characteristic $M$ and the income processes $\left(e^{i}\right)$ such that individual marginal valuation is the same as in the original equilibrium with heterogeneous beliefs. In other words, we proved that modulo a feasible modification of the individual incomes (i.e. $\sum_{i=1}^{N} \bar{e}^{i}=\sum_{i=1}^{N} e^{i}$ ) the initial equilibrium price process and trading volumes remain equilibrium price process and trading volumes in a homogeneous beliefs setting. The positive process $M$ can then be interpreted as a consensus characteristic. In particular, if there is no heterogeneity, i.e. if all the investors have the same belief represented by $M^{i}=\tilde{M}$ for all $i$, we obtain $M=\tilde{M}$ and there is no transfer nor optimal allocations modification (i.e. $\bar{e}^{i}=e^{i}$ and $\bar{y}^{i}=y^{*^{i}}$ for all $i$ ).

\footnotetext{
${ }^{3}$ This condition was introduced by Calvet et al. (2002). It would be equivalent to impose that the Lagrange multipliers are the same for each investor in both equilibria. As a consequence, the representative agent utility function will also be the same in both equilibria.
} 
Once the result on beliefs aggregation is achieved, it is easy to construct as in the standard case, a representative agent, i.e. an expected utility maximizing aggregate investor, representing the economy in equilibrium. As in the standard case, for $\alpha \in\left(\mathbb{R}_{+}^{*}\right)^{N}$, we introduce the function

$$
u_{\alpha}(t, x)=\max _{\sum_{i=1}^{N} x_{i} \leq x} \sum_{i=1}^{N} \frac{1}{\alpha_{i}} u_{i}\left(t, x_{i}\right) .
$$

Proposition 2.2. Consider an interior equilibrium $\left(q^{*},\left(y^{*^{i}}\right)\right)$ relative to the beliefs $\left(M^{i}\right)$ and the income processes $\left(e^{i}\right)$ and let $\left(\lambda_{i}\right)$ denote the Lagrange multipliers associated to this equilibrium. There exists a consensus investor defined by the normalized von Neumann-Morgenstern utility function $u_{\lambda}$ and the consensus characteristic $M$ of Proposition 2.1, in the sense that the portfolio $e^{*}$ maximizes his expected utility $E^{P}\left[\sum_{t=0}^{T} M_{t} u_{\lambda}\left(c_{t}\right)\right]$ under the market budget constraint $E^{P}\left[\sum_{t=0}^{T} q_{t}^{*}\left(c_{t}-e_{t}^{*}\right)\right] \leq 0$.

Remark that the consensus investor utility function is constructed as usual with the Lagrange multipliers of the initial equilibrium. In the specific case of linear risk tolerance utility functions we obtain the following explicit expressions for the consensus characteristic. Recall that the risk-tolerance of agent $i$ is defined by $T_{i}(t, y)=-\frac{u_{i}^{\prime}}{u_{i}^{\prime \prime}}(t, y)$.

Example 2.3. 1. If the individual utility functions are of exponential type, i.e. if $-\frac{u_{i}^{\prime}(t, x)}{u_{i}^{\prime \prime}(t, x)}=\theta_{i}>0$, then $u^{\prime}(t, x)=a e^{-x / \bar{\theta}}$ for $a=e^{e_{0}^{*} / \bar{\theta}}$ and $^{4}$

$$
M=\prod_{i=1}^{N}\left(M^{i}\right)^{\theta_{i} / \bar{\theta}}=\mathcal{E}_{0}^{\theta \cdot}\left(M^{\cdot}\right) .
$$

2. If the individual utility functions are of logarithmic or power type, i.e. such that $-\frac{u_{i}^{\prime}(t, x)}{u_{i}^{\prime \prime}(t, x)}=\theta_{i}+\eta x$ for $\eta \neq 0$, then $u^{\prime}(e)=b(\bar{\theta}+\eta e)^{-\frac{1}{\eta}}$ for $b=\left(\bar{\theta}+\eta e_{0}\right)^{\frac{1}{\eta}}$

\footnotetext{
${ }^{4}$ For given families of positive real numbers $\left(x_{i}\right)_{i=1, \cdots, n}$ and $\left(\theta_{i}\right)_{i=1, \cdots, n}$, we denote by $\bar{\theta}$ the sum $\sum_{i=1}^{n} \theta_{i}$, by $\mathcal{E}_{k}^{\theta}\left(x\right.$.) the weighted $k$-average defined for $k \in \mathbb{Z} \backslash\{0\}$ by $\mathcal{E}_{k}^{\theta}(x$.) $\equiv$ $\left[\sum_{i=1}^{n} \frac{\theta_{i}}{\theta} x_{i}^{k}\right]^{\frac{1}{k}}$ and we let $\mathcal{E}_{0}^{\theta}(x.) \equiv \prod_{i=1}^{n} x_{i}^{\frac{\theta_{i}}{\theta}}$ denote the geometric average. We denote by $\mathbf{1}$ the vector of $\mathbb{R}^{n}$ whose coordinates are all equal to 1 . With these notations, $\mathcal{E}_{k}^{\mathbf{1}}(x$.) is then the equally weighted $k$-average of the $x_{i}$.
} 
and

$$
\begin{aligned}
& M=\left[\sum_{i=1}^{N} \gamma_{i}\left(M^{i}\right)^{\eta}\right]^{\frac{1}{\eta}}=\mathcal{E}_{\eta}^{\lambda^{-\eta}}\left(M^{\cdot}\right) \\
&=\left[\sum_{i=1}^{N} \tau_{i}\left(M^{i}\right)^{-\eta}\right]^{-\frac{1}{\eta}}=\mathcal{E}_{-\eta}^{T .\left(t, y^{*}\right)}\left(M^{\cdot}\right) \\
& \text { for } \gamma_{i}=\frac{\lambda_{i}^{-\eta}}{\sum_{j=1}^{N} \lambda_{j}^{-\eta}} \text { and } \tau_{i}=\frac{T_{i}\left(t, y^{*^{i}}\right)}{\sum_{j=1}^{N} T_{j}\left(t, y^{*^{i}}\right)} .
\end{aligned}
$$

Notice that the consensus characteristic $M$ is a martingale (i.e. the density process of a given probability) only when $\eta=1$ (logarithmic case). It is a supermartingale when $\eta<1$, and a submartingale when $\eta>1$.

It is interesting to notice in Example 2.3 that for all utility functions in the classical class of linear risk tolerance utility functions, the consensus characteristic is obtained as a weighted average of the individual subjective beliefs, the weights being given by the individual risk tolerances. We assumed a linear risk tolerance and the same coefficient of risk aversion for all the agents and it would be interesting to have similar formulas when the agents have different cautiousness parameters $\eta_{i}$. In this case, and even for more general utility functions, it is easy to see that the consensus characteristic can still be considered as an average of the individual beliefs, however the formulas are intractable.

The process $M$ represents a consensus characteristic, however, except in the logarithmic case, it fails to be a martingale. Consequently, it can not be interpreted as a belief, i.e. the density process of a given probability measure. It is easy to see that it is not possible in general to recover the consensus characteristic as a martingale, as soon as we want the equilibrium price to remain the same and the optimal allocations in the equivalent equilibrium to be feasible, in the sense that they still add up to $e^{*}$ (even if we do not impose the invariance of individual marginal valuation).

This means that in the general case, there is a bias induced by the aggregation of the individual probabilities into a consensus probability.

Proposition 2.4. Consider an interior equilibrium price process $q^{*}$ relative to the beliefs $\left(M^{i}\right)$, and the income processes $\left(e^{i}\right)$. There exists a positive martingale process $\bar{M}$ with $\bar{M}_{0}=1$, and a predictable positive process $B$ with $B_{0}=1$ such that

$$
\bar{M}_{t} B_{t} u^{\prime}\left(t, e_{t}^{*}\right)=q^{*}
$$


The process $B$ is given by

$$
B_{t}=B_{t-1} \frac{E_{t-1}\left[M_{t}\right]}{M_{t-1}}, \quad B_{0}=1
$$

and can be thought of as a discount factor.

The process $B$ represents an aggregation bias. It satisfies $\frac{B_{t}}{B_{t-1}}=\frac{E_{t-1}\left[M_{t}\right]}{M_{t-1}}$ and measures the "distance" from martingality of the consensus characteristic $M$. If all investors share the same beliefs, then $B \equiv 1$. Otherwise, the process $B$ leads to a (possibly negative) discount of utility from future consumption. We shall interpret the presence and the properties of this "discount factor" below, after Example 2.5.

As mentioned before, our construction is different from Calvet et al. (2004). In both constructions, a degree of freedom is needed. In the representative agent construction of Calvet et al. (2004), the degree of freedom is given by an adjustment of the aggregate wealth while our degree of freedom is given by the introduction of a discount factor, that is easy to interpret and that will disappear in the CCAPM formula. Furthermore, the introduced discount factor does not impact as in Calvet et al. (2004) the construction of the consensus belief.

We shall see now that the process $\bar{M}$ corresponds to a (weighted) average of the individual beliefs and that the discount factor $B$ is directly related to the dispersion of the individual beliefs. We first consider two simple and enlightening examples.

Suppose that $\log \frac{e_{t+1}^{*}}{e_{t}^{*}}$ has a normal conditional distribution under $P$ and under $Q_{i}$, more precisely suppose that, under $P, \log \frac{e_{t+1}^{*}}{e_{t}^{*}} \sim_{F_{t}} \mathcal{N}(m, \sigma)$ and that under $Q_{i}, \log \frac{e_{t+1}^{*}}{e_{t}^{*}} \sim_{F_{t}} \mathcal{N}\left(\mu_{i}, \sigma\right)$ and that all the utility functions are exponential, i.e. $u_{i}^{\prime}(t, x)=e^{-\frac{x}{\theta_{i}}}$. We have

$$
\frac{M_{t+1}^{i}}{M_{t}^{i}}=\exp -\frac{\mu_{i}^{2}-m^{2}+2 \log \frac{e_{t+1}^{*}}{e_{t}^{*}}\left(m-\mu_{i}\right)}{2 \sigma^{2}}
$$

and consequently

$$
\begin{aligned}
\frac{M_{t+1}}{M_{t}} & =\exp -\frac{\sum_{i=1}^{N} \frac{\theta_{i}}{\bar{\theta}} \mu_{i}^{2}-m^{2}+2 \log \frac{e_{t+1}^{*}}{e_{t}^{*}}\left(m-\sum_{i=1}^{N} \frac{\theta_{i}}{\bar{\theta}} \mu_{i}\right)}{2 \sigma^{2}} \\
& =\frac{\bar{M}_{t+1}}{\bar{M}_{t}} \frac{B_{t+1}}{B_{t}}
\end{aligned}
$$


with $^{5}$

$$
\begin{aligned}
\frac{\bar{M}_{t+1}}{\bar{M}_{t}} & =\exp -\frac{\left[\mathcal{E}_{1}^{\theta \cdot}(\mu .)\right]^{2}-m^{2}+2 \log \frac{e_{t+1}^{*}}{e_{t}^{*}}\left(m-\mathcal{E}_{1}^{\theta \cdot}(\mu .)\right)}{2 \sigma^{2}} \\
\frac{B_{t+1}}{B_{t}} & =\exp -\frac{\sum_{i=1}^{N} \frac{\theta_{i}}{\bar{\theta}} \mu_{i}^{2}-\left(\sum_{i=1}^{N} \frac{\theta_{i}}{\bar{\theta}} \mu_{i}\right)^{2}}{2 \sigma^{2}} \\
& =\exp -\frac{\operatorname{Var}^{\theta \cdot}(\mu .)}{2 \sigma^{2}} .
\end{aligned}
$$

If we let $Q$ denote the probability measure with density process $\bar{M}$ with respect to $P$, we have under $Q, \log \frac{e_{t+1}^{*}}{e_{t}^{*}} \sim_{F_{t}} \mathcal{N}\left(\mathcal{E}_{1}^{\theta} \cdot(\mu),. \sigma\right)$. The aggregate belief $\bar{M}$ corresponds then clearly to a weighted average belief, the weights being given by the individual risk tolerances. The discount factor $\frac{B(t+1)}{B(t)}$ is directly related to $\operatorname{Var}^{\theta} \cdot(\mu$. $)$ the weighted variance of the $\mu_{i}$ with the same weights and the process $B$ can be interpreted as a measure of beliefs dispersion.

With the same utility functions and if we now assume that under $P, \log \frac{e_{t+1}^{*}}{e_{t}^{*}} \sim_{F_{t}}$ $\mathcal{N}(\mu, \sigma)$ and that under $Q_{i}, \log \frac{e_{t+1}^{*}}{e_{t}^{*}} \sim_{F_{t}} \mathcal{N}\left(\mu, \sigma_{i}\right)$, we obtain with the same notations that under $Q, \log \frac{e_{t+1}^{*}}{e_{t}^{*}} \sim_{F_{t}} \mathcal{N}\left(\mu, \mathcal{E}_{-2}^{\theta .}(\sigma).\right)$ which can still be interpreted as an average belief (the variance under $Q$ is an average of the variances under $Q_{i}$ ). Furthermore, $\frac{B(t+1)}{B(t)}=\frac{\mathcal{E}_{-2}^{\theta}(\sigma)}{\mathcal{E}_{0}^{\theta}(\sigma)}$ and a simple second order Taylor ${ }^{6}$ expansion gives us $\frac{B(t+1)}{B(t)} \sim 1-\frac{\operatorname{Var}^{\theta \cdot}\left(\sigma_{.}\right)}{\mathcal{E}_{1}^{\theta \cdot}(\sigma \cdot)^{2}}$ when the dispersion of the $\sigma_{i}$ is sufficiently small. Once again, the process $B$ is direcly related to beliefs dispersion and its distance from 1 increases with beliefs dispersion.

More generally, with HARA utility functions, it is easy to see using similar Taylor approximations that when beliefs dispersion is sufficiently small, $\bar{M}$ is equal, to the first order, to a risk-tolerance weighted arithmetic average of the $M^{i}$ and that the growth rate of $B$ is, at the second order, proportional to the dispersion of the $M^{i}$.

We are now able to explicitly compute the process $B$ and to determine whether it is increasing, decreasing, smaller or greater than one.

\footnotetext{
${ }^{5}$ For given families of positive real numbers $\left(x_{i}\right)_{i=1, \ldots, n}$ and $\left(\theta_{i}\right)_{i=1, \ldots, n}$, we denote by $\operatorname{Var}^{\theta} \cdot\left(x\right.$.) the weighted variance $\operatorname{Var}^{\theta} \cdot(x)=.\sum_{i=1}^{n} \frac{\theta_{i}}{\theta}\left[x_{i}-\mathcal{E}_{1}^{\theta}\left(x_{i}\right)\right]^{2}$.

${ }^{6}$ Note that straightforward expansions give us for $\operatorname{Var}^{\theta} \cdot(x$.) small enough, the following approximations $\mathcal{E}_{k}^{\theta \cdot}(x.) \sim \mathcal{E}_{1}^{\theta \cdot}(x)+.\frac{k-1}{2} \frac{\operatorname{Var}^{\theta \cdot}(x .)}{\mathcal{E}_{1}^{\theta}(x .)}$.
} 
Example 2.5. For general power utility functions, the process $B$ satisfies

$$
\frac{B_{t}}{B_{t-1}}=\frac{E_{t-1}\left[\mathcal{E}_{\eta}^{\gamma} \cdot\left(M_{\dot{t}}\right)\right]}{\mathcal{E}_{\eta}^{\gamma \cdot}\left(M_{t-1}\right)}
$$

so that by Minkowski's Lemma, $B$ is nondecreasing, greater than 1 (resp. nonincreasing, lower than 1) if $\eta \geq 1$ (resp. $\eta \leq 1$ ).

For logarithmic utility functions, $B \equiv 1$.

In the exponential case, the process $B$ satisfies

$$
\frac{B_{t}}{B_{t-1}}=\frac{E_{t-1}\left[\mathcal{E}_{0}^{\theta \cdot}\left(M_{t}\right)\right]}{\mathcal{E}_{0}^{\theta \cdot}\left(M_{t-1}\right)}
$$

so that by Hölder's Lemma, $B$ is nondecreasing, greater than 1 .

The interpretation is the following. The parameter $\eta$ is a cautiousness parameter. When there is more risk involved, depending on whether the investor is cautious or not, that is to say, depending on whether $\eta<1$ or $\eta>1$, it can be shown that the investor will reduce or increase current consumption with respect to future consumption. For instance, for $\eta<1$, the investor is cautious and increases current consumption acting as if his/her utility was discounted by a positive discount rate. The converse reasoning leads to a negative discount rate if $\eta>1$. Now in our context with heterogeneous beliefs, a possible interpretation consists in considering the dispersion of beliefs as a source of risk thereby leading for the representative agent to a discount factor associated to a positive or negative discount rate depending on whether $\eta<1$ or $\eta>1$. This interpretation of beliefs heterogeneity as a source of risk is compatible with the empirical findings of Cragg and Malkiel (1982) who studied the relationship between ex post returns and various measures of risk and found that the measure that performed best was a measure of divergence of opinion about the asset returns.

To summarize, we have pointed out through previous propositions two distinct effects of the introduction of beliefs heterogeneity on the equilibrium price.

There is first a change of probability effect from $P$ to the new common probability $Q$, whose density is given by $\bar{M}$. This aggregate probability can be seen as a weighted average of the individual subjective probabilities. The weights of this average, in the case of linear risk tolerance utility functions, are given by the individual risk tolerances. The second effect is represented by an "aggregation bias", which is predictable and takes the form of a discount factor. We have seen 
on simple examples that this discount factor is directly related to beliefs dispersion. We are able, for linear risk tolerance utility functions, to determine if it is associated to a positive or negative discount rate. Modulo this discount factor, the equilibrium (state) price (density) is the same as in an economy in which all agents (or the representative agent) would share the same subjective belief $Q$. Remark that the interpretation of beliefs heterogeneity as a source of risk is only related to the second effect. The relative importance of these effects is discussed in detail in Section 4.

We shall now use our construction of a representative consumer to analyze the impact of heterogeneity of beliefs on the equilibrium properties.

\subsection{Adjusted CCAPM, Risk Premium and Risk Free Rate}

In this subsection, we wish to compare the equilibrium characteristics (CCAPM formula, risk premium, risk free rate) in the heterogeneous beliefs setting and in the standard setting. We shall consider as the standard setting an equilibrium where the beliefs are homomogeneous and given by the objective probability $P$, and where the representative agent utility function is given by ${ }^{7} u_{\lambda}$ with the same $\left(\lambda_{i}\right)$ as in our heterogeneous beliefs setting ${ }^{8}$.

We suppose the existence of a riskless asset with price process $S^{0}$ such that $S_{0}^{0}=1$ and $S_{t}^{0}=\prod_{s=1}^{t}\left(1+r_{s}^{f}\right)$ for some predictable risk free rate process $r^{f}$. We consider a risky asset with positive price process $S$ and associated rate of return between date $t$ and $(t+1)$ denoted by $R_{t+1} \equiv \frac{S_{t+1}}{S_{t}}-1$. In such a context, since $q^{*} S$ is a $P^{*}$-martingale, we obtain, as in the classical case (see the Appendix),

$$
E_{t}^{P}\left[R_{t+1}\right]-r_{t+1}^{f}=-\operatorname{cov}_{t}^{P}\left[\frac{q_{t+1}^{*}}{E_{t}^{P}\left[q_{t+1}^{*}\right]}, R_{t+1}\right]
$$

\footnotetext{
${ }^{7}$ We recall that for $\alpha \in\left(\mathbb{R}_{+}^{*}\right)^{N}, u_{\alpha}(t, x) \equiv \max _{\sum_{i=1}^{N} x_{i} \leq x} \sum_{i=1}^{N} \frac{1}{\alpha_{i}} u_{i}\left(t, x_{i}\right)$.

${ }^{8}$ This is in particular the case when the standard setting equilibrium has the same Lagrange multipliers (or equivalently the same marginal valuations at date 0 or the same initial individual equilibrium consumptions) as in the heterogeneous beliefs framework, or without restriction on the Lagrange multipliers when investors have linear risk tolerance utility functions.
} 
Now, since $q_{t}^{*}=\bar{M}_{t} B_{t} u^{\prime}\left(t, e_{t}^{*}\right)$, with $B$ predictable, Equation (2.2) can be written

$$
\begin{aligned}
E_{t}^{P}\left[R_{t+1}\right]-r_{t+1}^{f} & =-\operatorname{cov}_{t}^{P}\left[\frac{\bar{M}_{t+1} B_{t+1} u^{\prime}\left(t+1, e_{t+1}^{*}\right)}{E_{t}^{P}\left[\bar{M}_{t+1} B_{t+1} u^{\prime}\left(t+1, e_{t+1}^{*}\right)\right]}, R_{t+1}\right] \\
& =-\operatorname{cov}_{t}^{P}\left[\frac{\bar{M}_{t+1} u^{\prime}\left(t+1, e_{t+1}^{*}\right)}{E_{t}^{P}\left[\bar{M}_{t+1} u^{\prime}\left(t+1, e_{t+1}^{*}\right)\right]}, R_{t+1}\right]
\end{aligned}
$$

so that the adjustment process $B$ plays no role in the CCAPM formula with heterogeneous beliefs. This adjusted CCAPM formula differs from the following standard formula

$$
E_{t}^{P}\left[R_{t+1}\right]-r_{t+1}^{f}=-\operatorname{cov}_{t}^{P}\left[\frac{u^{\prime}\left(t+1, e_{t+1}^{*}\right)}{E_{t}^{P}\left[u^{\prime}\left(t+1, e_{t+1}^{*}\right)\right]}, R_{t+1}\right]
$$

only through the change of probability from $P$ to the consensus probability $Q$. This implies that only the change of probability has an impact on the difference of the risk premium in the standard and in the heterogeneous beliefs setting.

The risk free rate $r^{f}$ is given, as in the classical case, by (see the Appendix)

$$
1+r_{t+1}^{f}=\frac{q_{t}^{*}}{E_{t}^{P}\left[q_{t+1}^{*}\right]}
$$

Now, since $q_{t}^{*}=\bar{M}_{t} B_{t} u^{\prime}\left(t, e_{t}^{*}\right)$, with $B$ predictable, we obtain

$$
\begin{aligned}
1+r_{t+1}^{f}[\text { het. }] & =\left(\frac{B_{t}}{B_{t+1}}\right) \frac{\bar{M}_{t} u^{\prime}\left(t, e_{t}^{*}\right)}{E_{t}^{P}\left[\bar{M}_{t+1} u^{\prime}\left(t+1, e_{t+1}^{*}\right)\right]} \\
& =\left(\frac{B_{t}}{B_{t+1}}\right)\left[1+r_{t+1}^{f}[\text { homogeneous under } Q]\right]
\end{aligned}
$$

where $r^{f}$ [homogeneous under $Q$ ] denotes the equilibrium risk free rate in a model where all investors share the same subjective probability belief $Q$. Both the change of probability and the discount factor have an impact on the risk free rate. The impact of the discount factor leads to a decrease (resp. increase) of the riskfree rate if $B$ is nondecreasing (resp. nonincreasing). This effect has a clear interpretation. A nonincreasing (resp. nondecreasing) discount factor $B$ corresponds to the fact that the representative agent "discounts" future consumption, which means that future consumption is less (resp. more) important for the representative agent, 
it is then natural to obtain a higher (resp. lower) equilibrium risk free rate. For instance, for power utility functions with $\eta \leq 1$, as well as for exponential utility functions, we have seen that the "discount factor" $B$ is nonincreasing and therefore contributes to a higher risk free rate.

The impact of the change of probability effect from $P$ to the consensus probability $Q$ depends on the sign of $E_{t}^{Q}\left[\frac{u^{\prime}\left(t+1, e_{t+1}^{*}\right)}{u^{\prime}\left(t, e_{t}^{*}\right)}\right]-E_{t}^{P}\left[\frac{u^{\prime}\left(t+1, e_{t+1}^{*}\right)}{u^{\prime}\left(t, e_{t}^{*}\right)}\right]$.

Through our aggregation procedure, we have then transformed the study of a model under heterogeneous subjective beliefs into the study of a model under homogeneous subjective beliefs. Indeed, the equilibrium risk premium formula under heterogeneous beliefs (Equation 2.3) is the same as in an economy where all investors would share the same probability belief, namely the consensus belief obtained through the aggregation procedure. The same is true of the risk free rate, modulo the "discount factor", and we have seen that we are able to determine the impact of the discount factor for classical utility functions. The next step consists in the study of the impact of a subjective belief on the equilibrium risk premium and risk free rate, which is the aim of the next section.

\section{Pessimism/doubt of the consensus belief}

We consider in this section a homogeneous beliefs model, the agents common subjective belief $\widehat{P}$ being possibly different from the objective probability $P$. We suppose that $\widehat{P}$ is equivalent to the objective probability $P$ and we denote by $\widehat{M}$ its density. We want to determine characteristics of the subjective belief $\widehat{P}$ that have an unambiguous impact on the risk premium and on the risk free rate, and, in relation with the risk premium puzzle (see Mehra-Prescott, 1985 or Kocherlakota, 1996 for a survey on the risk premium puzzle) and the risk free rate puzzle (Weil, 1989), which preferrably lead to an increase of the risk premium and a decrease of the risk free rate. In particular, we wish to analyze how pessimism/doubt of the subjective probability may affect the equilibrium risk premium and risk free rate.

In order to analyze the impact of the subjective belief on the value of the risk premium, we need to be aware of the fact that the possible returns $R=\left(R_{t}\right)_{t=1}^{T}$ obtained in equilibrium are not the same in the standard (objective belief) setting and in the subjective belief settings. What exactly is to be compared?

As in the classical CCAPM, we consider an asset, whose returns $R_{t+1}$ between date $t$ and date $t+1$ are perfectly correlated with $e_{t+1}^{*}$ from date $t$ point of view. More precisely, we consider a return process such that $R_{t+1}=k_{t} e_{t+1}^{*}+b_{t}$ for some 
$F_{t}$-measurable positive random variables $k_{t}$ and $b_{t}$. Notice that such an asset exists by market (dynamic) completeness. The risk premium for this asset in the standard objective belief setting is given by

$$
E_{t}^{P}\left[R_{t+1}\right]-r_{t+1}^{f}=-\operatorname{cov}_{t}^{P}\left[\frac{u^{\prime}\left(e_{t+1}^{*}\right)}{E_{t}^{P}\left[u^{\prime}\left(e_{t+1}^{*}\right)\right]}, k_{t} e_{t+1}^{*}\right] .
$$

For any positively homogeneous risk measure $\rho$, the market price of risk i.e., the ratio between the risk premium and the "level of risk" is given by

$$
M P R_{t}^{o b j}\left(R_{t+1}\right)=-\frac{1}{\rho\left(e_{t+1}^{*}\right)} \operatorname{cov}_{t}^{P}\left[\frac{u^{\prime}\left(e_{t+1}^{*}\right)}{E_{t}^{P}\left[u^{\prime}\left(e_{t+1}^{*}\right)\right]}, e_{t+1}^{*}\right] .
$$

Since this quantity does not depend upon $k_{t}$ and $b_{t}$, we shall denote it by $M P R_{t}^{o b j}\left(e_{t+1}^{*}\right)$. We obtain analogously in the subjective belief setting under $\widehat{P}$ that

$$
\operatorname{MPR}_{t}^{\operatorname{subj}(\widehat{P})}\left(e_{t+1}^{*}\right)=-\frac{1}{\rho\left(e_{t+1}^{*}\right)} \operatorname{cov}_{t}^{P}\left[\frac{\widehat{M}_{t+1} u^{\prime}\left(e_{t+1}^{*}\right)}{E_{t}^{P}\left[\widehat{M}_{t+1} u^{\prime}\left(e_{t+1}^{*}\right)\right]}, e_{t+1}^{*}\right] .
$$

In order to compare the market price of risk in both settings, we are then led to compare $-\operatorname{cov}_{t}^{P}\left[\frac{u^{\prime}\left(e_{t+1}^{*}\right)}{E_{t}^{P}\left[u^{\prime}\left(e_{t+1}^{*}\right)\right]}, e_{t+1}^{*}\right]$ with $-\operatorname{cov}_{t}^{P}\left[\frac{\widehat{M}_{t+1} u^{\prime}\left(e_{t+1}^{*}\right)}{E_{t}^{P}\left[\widehat{M}_{t+1} u^{\prime}\left(e_{t+1}^{*}\right)\right]}, e_{t+1}^{*}\right]$. The subjective belief setting leads to a higher market price of risk if and only if

$$
\frac{E_{t}^{\widehat{P}}\left[u^{\prime}\left(e_{t+1}^{*}\right) e_{t+1}^{*}\right]}{E_{t}^{\widehat{P}}\left[u^{\prime}\left(e_{t+1}^{*}\right)\right]} \leq \frac{E_{t}^{P}\left[u^{\prime}\left(e_{t+1}^{*}\right) e_{t+1}^{*}\right]}{E_{t}^{P}\left[u^{\prime}\left(e_{t+1}^{*}\right)\right]} .
$$

Abel (2002) studies a similar problem. He considers a return process $R_{t}$ generated by an asset whose dividends are given by $e_{t}^{*}$. In Abel's framework $\left(\frac{e_{t+1}^{*}}{e_{t}^{*}}\right.$ i.id. and isoelastic utility functions), such a return process satisfies our condition $R_{t+1}=k_{t} e_{t+1}^{*}+b_{t}$ for some $F_{t}$-measurable positive random variables $k_{t}$ and $b_{t}$. Abel's definition of the risk premium is slightly different, since it is equal to the ratio between the expected return of the considered asset and the riskfree return, instead of the difference between these two quantities. However, the probability measures leading to a higher risk premium (in Abel's sense) are those satisfying Inequality (3.1). 
To summarize what we have just seen, the subjective probability measures $\widehat{P}$ leading to an increase of the market price of risk (compared to the standard objective belief setting) are characterized by Inequality (3.1) or equivalently by the fact that

$$
\operatorname{cov}_{t}^{P_{u}}\left(\widehat{M}_{t+1}, e_{t+1}^{*}\right) \leq 0
$$

where $\frac{d P_{u}}{d P}$ is given (up to a constant) by $u^{\prime}\left(t+1, e_{t+1}^{*}\right)$ and $\widehat{M}_{t+1}=E\left[\frac{d \widehat{P}}{d P} \mid F_{t+1}\right]$. The subjective belief setting leads to a higher market price of risk when the subjective probability is negatively correlated with the total wealth under some probability and it is natural to interpret this property as a form of pessimism. Moreover, as seen in the previous section, the subjective probability measures $\widehat{P}$ leading to a decrease of the risk free rate are characterized by

$$
E_{t}^{\widehat{P}}\left[\frac{u^{\prime}\left(t+1, e_{t+1}^{*}\right)}{u^{\prime}\left(t, e_{t}^{*}\right)}\right] \geq E_{t}^{P}\left[\frac{u^{\prime}\left(t+1, e_{t+1}^{*}\right)}{u^{\prime}\left(t, e_{t}^{*}\right)}\right]
$$

or equivalently by

$$
\operatorname{cov}_{t}^{P}\left[\widehat{M}_{t+1}, u^{\prime}\left(e_{t+1}^{*}\right)\right] \geq 0,
$$

which also corresponds to some form of pessimism. We shall introduce more formally different notions of pessimism in the next section. Note that even if this section is in the same spirit as Abel (2002), our framework and definitions are different. Indeed, Abel (2002) first characterizes pessimism by the first-order stochastic dominance and then introduces the concept of uniform pessimism characterized by a stronger form of dominance ${ }^{9}$. Abel (2002) proves then that uniform pessimism increases the risk premium, when agents have power utility functions. Jouini-Napp (2005) showed that this is no longer the case when uniform pessimism is replaced by pessimism (in Abel's sense) or when the class of utility functions is enlarged to the whole class of concave and nondecreasing functions. In the next section we consider a dynamic concept of pessimism, that is particularly well adapted to the beliefs aggregation problem. Abel (2002) also introduces the concept of doubt based on second-order stochastic dominance. In the next Section we shall propose another definition that will appear in Section 4 as much more

\footnotetext{
${ }^{9}$ Abel (2002) defines a pessimistic (resp. uniformly pessimistic) probability measure (with respect to $e$ ) as a probability measure $\widehat{P}$ such that, for all $x, \widehat{P}[e \leq x] \geq P[e \leq x]$ (resp. $\widehat{P}[e \leq x]=P[e \leq k x]$ for some $k>1)$.
} 
tractable in order to analyze how individual doubt is captured at the aggregate level.

\subsection{Doubt and first-order pessimism}

Before introducing our concept of pessimism, we recall the following concept of conditional comonotonicity, that generalizes the classical unconditional comonotonicity concept. Two random variables $x$ and $z$ are said to be comonotonic conditionally to $F_{t}$ if the conditional law of $(x, z)$ with respect to $F_{t}$ has a comonotonic support ${ }^{10}$. It can be shown that it is equivalent to assuming that $x$ and $z$ are conditionally to $F_{t}$ nonincreasing functions of some third random variable.

Definition 3.1. We say that a probability $\widehat{P}$ on $(\Omega, F, P)$ equivalent to $P$, with density process $\left(\widehat{M}_{t}\right)$ is pessimistic (with respect to $e^{*}$ ) if for all $t, \widehat{M}_{t+1}$ and $-e_{t+1}^{*}$ are comonotonic conditionally to $F_{t}$.

A pessimistic probability is such that its density $\widehat{M}_{t+1}$ at date $(t+1)$ decreases with $e_{t+1}^{*}$ conditionally to $F_{t}$ for all $t$; this means that conditionally to date $t$ information, it puts more weight on states of nature where $e_{t+1}^{*}$ is low, which clearly corresponds to a notion of pessimism.

For instance, if the information structure is described by a tree and if, for a given node $\varpi$, the transition probability of $\widehat{P}$ (resp. $P$ ) between $\varpi$ and its immediate successors is given by $\widehat{\pi}_{\varpi}$ (resp. $\pi_{\varpi}$ ), then $\widehat{P}$ is pessimistic (with respect to $\left.e^{*}\right)$ if, for each $t$ and each date- $t$ node $\varpi$, the transition density $\frac{\widehat{\pi}_{\varpi}}{\pi_{\varpi}}$ is comonotonic with $-e_{t+1}^{*}$ on the set of successors of $\varpi$. In particular this will be the case if $\frac{\widehat{\pi}_{\varpi}}{\pi_{\varpi}}$ decreases with $e_{t+1}^{*}$.

If $F_{t+1}$ is generated by $e_{t+1}^{*}$ and $\frac{e_{t+1}^{*}}{e_{t}^{*}} \sim_{F_{t}} \mathcal{N}(\mu, \sigma)$ (resp. $\left.\mathcal{N}(\widehat{\mu}, \widehat{\sigma})\right)$ under $P$ (resp. $\widehat{P}$ ) then $\widehat{P}$ is pessimistic (with respect to $e^{*}$ ) between $t$ and $(t+1)$ if and only if $\mu \geq \widehat{\mu}$ and $\sigma=\widehat{\sigma}$. The same result holds for lognormal distributions. This result remains valid if $\mu$ and $\sigma$ are $F_{t}$-measurable random variables and if the distributions are replaced by conditional distributions.

If $\left(e_{t}^{*}\right)$ follows a Cox-Ross-Rubinstein binomial process with "returns" at each period denoted by $u$ and $d$ and associated transition probabilities $\pi_{u}$ and $\pi_{d}=$ $1-\pi_{u}$ (resp. $\widehat{\pi}_{u}$ and $\widehat{\pi}_{d}=1-\widehat{\pi}_{u}$ ) under $P$ (resp. $\widehat{P}$ ), then $\widehat{P}$ is pessimistic if and

\footnotetext{
${ }^{10} \mathrm{~A}$ subset $A \subset R^{2}$ is said to be comonotonic if for all $\left((x, y),\left(x^{\prime}, y^{\prime}\right)\right) \in A \times A$ we have $(x-y)\left(x^{\prime}-y^{\prime}\right) \geq 0$.
} 
only if $\pi_{u} \geq \widehat{\pi}_{u}$. As previously, the result remains valid if we introduce a time and state dependence for $u, d, \pi_{u}$ and $\widehat{\pi}_{u}$ as long as $u(t)$ and $d(t)$, the returns between $t$ and $(t+1)$ are $F_{t}$-measurable.

In a static setting, Jouini-Napp (2005) introduced a concept of second-order pessimism, characterized by the fact that Inequality (3.1) is satisfied for all nondecreasing and concave utility functions.

In particular, it is shown that if $\widehat{P}$ is second-order pessimistic with respect to $e^{*}$ then $E^{\widehat{P}}\left[e^{*} \mid e^{*} \leq x\right] \leq E^{P}\left[e^{*} \mid e^{*} \leq x\right]$ for all $x$, which means that the notion of second order pessimism is another way of expressing the fact that $\widehat{P}$ "puts more weight" than $P$ on the bad states of the world and corresponds then naturally to a concept of pessimism.

It is also shown that if a second order pessimistic probability measure $\widehat{P}$ satisfies $E^{\widehat{P}}\left[e^{*}\right]=E^{P}\left[e^{*}\right]$, then $\operatorname{Var}^{\widehat{P}}\left[e^{*}\right] \geq \operatorname{Var}^{P}\left[e^{*}\right]$. This permits one to relate this concept of pessimism to the concept of doubt introduced by Abel (2002).

This notion of second order pessimism appears as hard to characterize from a practical point of view. In a static setting, Jouini-Napp (2005) introduced a subset of the second order pessimistic probability measures that are simple to describe and that clearly exhibit doubt ${ }^{11}$. A natural generalization of this class of measures in a dynamic framework leads to the following definition in the case where the conditional distribution of $e_{t+1}^{*}$ is symmetric.

Definition 3.2. Suppose that for all $t$, the $F_{t}$-conditional distribution of $e_{t+1}^{*}$ under $P$ is symmetric with respect to $E_{t}\left[e_{t+1}^{*}\right]$. We say that a probability $\widehat{P}$ on $(\Omega, F, P)$ equivalent to $P$, with density process $\left(\widehat{M}_{t}\right)$ exhibits doubt (resp. overconfidence) between date $t$ and $(t+1)$ (with respect to $e^{*}$ ) if for all $t, \widehat{M_{t+1}}(\omega)=$ $f_{t}\left(\omega, e_{t+1}^{*}(\omega)-E_{t}\left[e_{t+1}^{*}\right](\omega)\right)$ where $f_{t}$ is $F_{t}$-measurable with respect to its first variable, even and nondecreasing (resp. nonincreasing) on $\mathbb{R}_{+}$with respect to its second variable.

This means that a probability measure, equivalent to $P$, exhibits doubt (resp. overconfidence) between date $t$ and $(t+1)$ if conditionally to date $t$ information, its density puts more (resp. less) weight on the tails and less (resp. more) weight on the center of the distribution. If $F_{t+1}$ is generated by $e_{t+1}^{*}$ and $\frac{e_{t+1}^{*}}{e_{t}^{*}} \sim_{F_{t}} \mathcal{N}(\mu, \sigma)$

\footnotetext{
${ }^{11}$ Suppose that $e^{*}$ has a symmetric distribution under $P$ with respect to $E^{P}\left[e^{*}\right]$. A probability $P^{\prime}$ on $(\Omega, F)$ equivalent to $P$, with density $M^{\prime}$ is said to exhibit doubt with respect to $e^{*}$ if $M^{\prime}$ is a function of $e^{*}$, symmetric with respect to $E^{P}\left[e^{*}\right]$ nonincreasing before $E^{P}\left[e^{*}\right]$ (and nondecreasing after).
} 
(resp. $\mathcal{N}(\widehat{\mu}, \widehat{\sigma}))$ under $P$ (resp. $\widehat{P})$, then $\widehat{P}$ exhibits doubt (with respect to $e^{*}$ ) between $t$ and $(t+1)$ if and only if $\mu=\widehat{\mu}$ and $\sigma \leq \widehat{\sigma}$.

If $\left(e_{t}^{*}\right)$ follows a trinomial process with "returns" at each period $u, m$ and $d$ and associated transition probabilities $\pi, 1-2 \pi$, and $\pi$ (resp. $\widehat{\pi}, 1-2 \widehat{\pi}$ and $\widehat{\pi}$ ) under $P$ (resp. $\widehat{P})$, then $\widehat{P}$ exhibits doubt if and only if $\pi \leq \widehat{\pi}$.

\subsection{Impact on the market price of risk and on the interest rate}

We prove that pessimism and doubt lead to a higher market price of risk.

Proposition 3.3. If the probability $\widehat{P}$ is pessimistic or exhibits doubt in the sense of the previous definitions, then for all $t$,

$$
\operatorname{MPR}_{t}^{\text {subj}(\widehat{P})}\left(e_{t+1}^{*}\right) \geq M P R_{t}^{o b j}\left(e_{t+1}^{*}\right)
$$

i.e., the market price of risk between $t$ and $(t+1)$ in the subjective belief setting under $\widehat{P}$ is greater than in the standard objective belief setting.

The interpretation is the following. In fact, the market price of risk subjectively expected is not modified by some pessimism. The reason why pessimism increases the objective expectation of the market price of risk is not that a pessimistic representative agent requires a higher market price of risk. He/She requires the same market price of risk but his/her pessimism leads him/her to underestimate the average rate of return of equity (leaving unchanged his/her estimation of the risk free rate). Thus the objective expectation of the equilibrium market price of risk is greater than the representative agent's subjective expectation, hence is greater than the standard market price of risk. The same interpretation holds for doubt. These results are consistent with the empirical findings of Cecchetti, Lam and Mark (2000) and Giordani and Söderlind (2003). In the first reference the authors prove that a model in which consumers exhibit pessimistic beliefs can better match sample moments of asset returns than can a rational expectations model. In the second reference the authors provide evidence of pessimism in investors forecasts.

As far as the risk free rate is concerned, we obtain the following result.

Proposition 3.4. $\quad$ 1. If the subjective probability belief $\widehat{P}$ exhibits pessimism and if the representative agent's utility function is concave and nondecreasing, then the equilibrium risk free rate is lower in the subjective belief setting than in the standard objective belief setting. 
2. If the subjective probability belief $\widehat{P}$ exhibits doubt and if the representative agent's utility function is nondecreasing, concave, with a convex derivative, then the equilibrium risk free rate is lower in the subjective belief setting than in the standard objective belief setting.

Hence, for a large class of utility functions including HARA utility functions, if there is doubt, the change of probability decreases the risk free rate. This result holds true if there is pessimism without restriction on the utility functions.

\subsection{Application to the heterogeneous beliefs model}

We adopt the same notations as above and we denote by $M P R_{t}^{\text {het }}\left(e_{t+1}^{*}\right)$ the market price of risk in the heterogeneous beliefs setting given by

$$
\operatorname{MPR}_{t}^{h e t}\left(e_{t+1}^{*}\right)=-\frac{1}{\rho\left(e_{t+1}^{*}\right)} \operatorname{cov}_{t}^{P}\left[\frac{\bar{M}_{t+1} u^{\prime}\left(e_{t+1}^{*}\right)}{E_{t}^{P}\left[\bar{M}_{t+1} u^{\prime}\left(e_{t+1}^{*}\right)\right]}, e_{t+1}^{*}\right] .
$$

We want to compare this quantity to the market price of risk in the standard setting, as well as the risk free rate in both settings. We have seen in the preceding section that the heterogeneous beliefs model can be analyzed in terms of an equivalent homogeneous beliefs model, the homogeneous belief being given by the consensus probability belief. In particular, we have seen that $\operatorname{MPR} R_{t}^{\text {het }}\left(e_{t+1}^{*}\right)=$ $M P R_{t}^{\operatorname{subj}(Q)}\left(e_{t+1}^{*}\right)$ where $Q$ denotes the consensus probability belief and $1+$ $r_{t+1}^{f}[$ heterogeneous $]=\left(\frac{B_{t}}{B_{t+1}}\right)\left[1+r_{t+1}^{f}[\right.$ homogeneous under $\left.Q]\right]$. An immediate application of our results on pessimism and doubt leads then to the following proposition.

Proposition 3.5. 1. If the consensus probability belief $Q$ exhibits pessimism and/or doubt then the market price of risk in the heterogeneous beliefs setting is higher than in the standard objective belief setting.

2. If the representative agent utility function is in the HARA class (i.e. $-\frac{u^{\prime}(t, x)}{u^{\prime \prime}(t, x)}=$ $\theta+\eta x)$ with a cautiousness parameter $\eta>1$ and if $Q$ exhibits pessimism and/or doubt then the equilibrium interest rate in the heterogeneous beliefs setting is lower than in the standard objective belief setting.

The last step of our analysis consists now in determining how pessimism and doubt at the aggregate level (i.e. on the consensus belief $Q$ ) can be inherited from the pessimism and doubt at the individual level (i.e. on the $Q_{i}$ ). 


\section{From individual to aggregate pessimism/doubt}

The aim of this subsection is to analyze how the properties of the individual beliefs are transferred to the consensus belief. In particular, since we have seen in the preceding section that a pessimistic consensus probability as well as a consensus probability which exhibits doubt leads to a higher market price of risk and lowers the risk free rate, we wish to explore which properties of the individual beliefs lead to a pessimistic consensus belief or to a consensus belief which exhibits doubt.

We start with the analysis of the notion of pessimism.

\subsection{From individual to aggregate pessimism}

Notice that there is no need for all investors to be pessimistic in order to obtain a pessimistic consensus belief, hence an increase of the market price of risk and a decrease of the risk free rate. Indeed, since the consensus probability corresponds to some average of the individual beliefs, it suffices that some average of the individual beliefs be pessimistic.

If all the agents have exponential utility functions, we have seen that $M=$ $\mathcal{E}_{0}^{\theta \cdot}\left(M^{\cdot}\right)$. The aggregate characteristic $M$ overweights the beliefs $M^{i}$ for which $\theta_{i}$ is greater than the average and underweights the beliefs $M^{i}$ for which $\theta_{i}$ is smaller than the average.

In order to enlighten our analysis, let us consider the case where $\frac{e_{t+1}^{*}}{e_{t}^{*}} \sim_{F_{t}}$ $\mathcal{N}(\mu, \sigma)$ under $P$, and $\frac{e_{t+1}^{*}}{e_{t}^{*}} \sim_{F_{t}} \mathcal{N}\left(\mu_{i}, \sigma\right)$ under $Q^{i}$. As in Section 2, we easily obtain that $\frac{e_{t+1}^{*}}{e_{t}^{*}} \sim_{F_{t}} \mathcal{N}\left(\mathcal{E}_{1}^{\theta}(\mu\right.$. $\left.), \sigma\right)$ under $Q$.

If all $\theta_{i}$ are equal, then $M=\mathcal{E}_{0}^{1}\left(M^{*}\right)$, which means that the consensus characteristic $M$ is an equally-weighted geometric average of the individual beliefs and the expected growth rate under the consensus characteristic appears as the equal weighted average of the individual subjective expected growth rates. The impact on the market price of risk is then simply given by the pessimism/optimism of the "equal-weighted average" investor. If the investors are on average pessimistic i.e. if $\mathcal{E}_{1}^{1}(\mu$. $) \leq \mu$ (resp. optimistic, i.e. if $\mathcal{E}_{1}^{1}(\mu$. $) \geq \mu$ ), then the market price of risk is higher (resp. lower) than in the standard setting - and the risk free rate is lower (resp. higher).

If the $\theta_{i}$ are different, the expected return under the consensus belief can be written as $\mathcal{E}_{1}^{\boldsymbol{\theta}}(\mu$. $)=\mathcal{E}_{1}^{\mathbf{1}}(\mu)+.\sum_{i=1}^{N}\left(\mu_{i}-\mathcal{E}_{1}^{\mathbf{1}}(\mu).\right)\left(\frac{\theta_{i}}{\bar{\theta}}-\frac{1}{N}\right)$. There are then two effects of beliefs heterogeneity on the equilibrium characteristics. The first effect 
is given as in the previous case by the average level of optimism/pessimism and the second effect is given by the "correlation" between risk tolerance/aversion and optimism/pessimism. If we assume for instance that the more risk tolerant investors are pessimistic (resp. optimistic) and the less risk tolerant investors are optimistic (resp. pessimistic), then the second effect increases (resp. reduces) the market price of risk. The intuition leads us to think that risk tolerance and optimism are positively correlated, which would induce a lower risk premium for assets with a higher dispersion. It remains to prove that this intuition is correct (or not). This could be done through behavioral or psychological empirical studies, and to our knowledge, this question is still open. This could also be done through the introduction in our model of a specific learning process which would lead to such a correlation, and this is left for future research.

Let us now assume that the utility functions are no longer exponential but of the power form $u^{\prime}(x)=\left(\theta_{i}+\eta x\right)^{-\frac{1}{\eta}}$. The consensus characteristic $M$ is now given by $\mathcal{E}_{\eta}^{\gamma \cdot}\left(M^{*}\right)$. Our aim is to exhibit a third effect and for this purpose, let us assume that all the agents are equally weighted in the definition of $M$ (no weights effect) and that there is no systematic bias in the average returns individual estimations (i.e. $\mathcal{E}_{1}^{\mathbf{1}}(\mu$. $)=\mu$ ). In order to simplify the analysis, we will even assume that the $\mu_{i}$ are symmetrically distributed around $\mu$. The equally weighted geometric average belief is then equal to the objective belief and the consensus belief is an equally weighted average of the individual beliefs. However, this last average is an $\eta$-average and not a geometric average and, contrarily to the exponential case, the consensus belief is not equal to the objective one. The consensus characteristic $M$ is such that $\frac{M_{t+1}}{M_{t}}=\left(\frac{1}{N} \sum_{i=1}^{N} \exp \frac{\eta}{2 \sigma^{2}}\left[-\left(\mu_{i}-\mu\right)^{2}-2\left(\mu-\mu_{i}\right)\left(\frac{e_{t+1}^{*}}{e_{t}^{*}}-\mu\right)\right]\right)^{\frac{1}{\eta}}$. This function is clearly symmetric with respect to $\mu$, increasing after $\mu$. Then the consensus probability $Q$ exhibits doubt and the market price of risk is higher than in the standard setting. It is easy to check that this doubt effect increases with the cautiousness parameter $\eta$. More precisely, if we denote by $Q^{\eta}$ (resp. $Q^{\eta^{\prime}}$ ) the consensus probability when all the agents have a cautiousness parameter $\eta$ (resp. $\eta^{\prime}$ ) then, for $\eta^{\prime} \geq \eta$, the density of $Q^{\eta^{\prime}}$ with respect to $Q^{\eta}$ is symmetric with respect to $\mu$, and increases after $\mu$. The probability $Q^{\eta^{\prime}}$ exhibits more doubt than $Q^{\eta}$ and the market price of risk is higher in the $\eta^{\prime}$ cautiousness framework than in the $\eta$ cautiousness framework.

We have shown in these examples that there are three possible effects when we aggregate individual heterogeneous beliefs into a consensus belief:

- an average effect: if the (equally weighted) average belief is pessimistic or 
optimistic, then the consensus belief will be influenced accordingly,

- a cautiousness effect: when the consensus belief is an $\eta^{\prime}$-average with given weights $^{12}$ and the objective probability is an $\eta$-average with the same weights, then there is a bias towards doubt if $\eta^{\prime} \geq \eta$ and a bias towards overconfidence if $\eta \geq \eta^{\prime}$.

- a relative weights effect: if the more risk tolerant investors are pessimistic (resp. optimistic) and the less risk tolerant investors are optimistic (resp. pessimistic), then the consensus belief will present a bias toward pessimism (resp. optimism).

The first effect is natural and does not necessitate specific developments. The second effect has been studied in a different framework by Gollier (2003) where the author analyzes the social impact of different exogeneous aggregation of beliefs procedures. With our notations, Gollier (2003) states in particular for some specific values of the cautiousness parameter $\eta$ that the $\eta^{\prime}$-average aggregation procedure is socially efficient if and only if $\eta^{\prime}=\eta$. When this condition is not satisfied, Gollier (2003) analyzes the impact of a disagreement increase depending on the location of this increase (in the tails or in the center of the distribution).

However, the Taylor expansions provided in Section 2 permit us to show that these distinct averages differ only by second order terms, when the beliefs dispersion is sufficiently small. Hence the second effect deserves specific attention only when the two other effects cancel out.

Abel (1989) studied a problem that is similar to ours in a specific framework. He imposes a normal distribution for the aggregate wealth and considers exponential utility functions with the same risk tolerance parameters $\theta_{i}$. Furthermore, it is assumed that the "average investor is rational", i.e. the geometric average belief does not exhibit any bias and is equal to the objective probability. Under these assumptions our three effects vanish and there is no impact on the market price of risk as defined in this paper. Abel (1989) is not interested in the impact of beliefs heterogeneity on the market price of risk but on the risk premium. The impact of beliefs heterogeneity on the risk premium is directly related to the impact on the risk free rate and Abel finds that beliefs heterogeneity leads to an increase of the risk premium.

In the following, we focus on the third and last effect and, for this purpose, we suppose that the equally-weighted average of the individual beliefs corresponds to

\footnotetext{
${ }^{12}$ i.e. when we have CRRA utility functions with a cautiousness parameter $\eta$.
} 
the objective probability $P$. We obtain the following result, without any specific assumption on the distributions of the growth rate of aggregate wealth.

Proposition 4.1. Assume that $-\frac{u_{i}^{\prime}(t, x)}{u_{i}^{\prime \prime}(t, x)}=\theta_{i}+\eta x>0$, and that

$$
\frac{\mathcal{E}_{\eta}^{\mathbf{1}}\left(M_{t+1}\right)}{E_{t}\left[\mathcal{E}_{\eta}^{\mathbf{1}}\left(M_{t+1}\right)\right]}=1 \quad t=0, . ., T
$$

If the pessimistic (resp. optimistic) agents have a risk tolerance higher (resp. lower) than the average, then the market price of risk in the heterogeneous beliefs setting is greater than in the standard setting.

Remark that the condition on the $\left(M^{i}\right)$ still means that the equally-weighted average of the individual beliefs corresponds to the objective probability $P$. However, the geometric average is replaced by the power $\eta$ average. It is possible to replace this condition by a geometric average condition (or an $\eta^{\prime}$-average with $\left.\eta^{\prime}<\eta\right)$ modulo an additional "cautiousness" effect. As mentioned previously, this effect is of the second order for small dispersions. Furthermore, this effect should, as in the examples above, lead to an additional increase of the market price of risk.

We can adopt the same approach to analyze how doubt/overconfidence at the aggregate level can be inherited from the doubt/overconfidence at the individual level.

\subsection{From individual to aggregate doubt}

In the case of exponential utility functions, we have seen that $M=\mathcal{E}_{0}^{\theta}\left(M^{\cdot}\right)$ and $M$ overweights the beliefs $M^{i}$ for which $\theta_{i}$ is greater than the average and underweights the beliefs $M^{i}$ for which $\theta_{i}$ is smaller than the average. For instance, suppose that under $P, \frac{e_{t+1}^{*}}{e_{t}^{*}} \sim_{F_{t}} \mathcal{N}(\mu, \sigma)$ and that under $Q_{i}, \frac{e_{t+1}^{*}}{e_{t}^{*}} \sim_{F_{t}} \mathcal{N}\left(\mu, \sigma_{i}\right)$. It is then easy to see that, as in Section $2, \frac{e_{t+1}^{*}}{e_{t}^{*}} \sim_{F_{t}} \mathcal{N}\left(\mu, \mathcal{E}_{-2}^{\theta}(\sigma).\right)$ under $Q$. There are then two effects of beliefs heterogeneity on the market price of risk. The first effect (the average effect) is given by the average level of doubt/overconfidence, measured by an equally weighted average of the individual levels of overconfidence $\frac{1}{\sigma_{i}^{2}}$. The second effect (relative weights effect) is given by the covariance between individual risk tolerance/aversion and the individual level of overconfidence. If we assume for instance that the more risk tolerant investors exhibit doubt and that 
the less risk tolerant investors exhibit overconfidence, then we get a higher (resp. lower) market price of risk. We could, as in the analysis of pessimism, exhibit a third "cautiousness" effect if we consider power utility functions.

As above, if we focus on the "relative weights effect" and suppose that the equally-weighted average of the individual beliefs corresponds to the objective probability $P$, we obtain the analog of Proposition 4.1.

Proposition 4.2. Assume that $-\frac{u_{i}^{\prime}(t, x)}{u_{i}^{\prime \prime}(t, x)}=\theta_{i}+\eta x$ (with $\eta$ possibly equal to zero) and for all $t, \frac{\mathcal{E}_{\eta}^{1}\left(M_{i+1}\right)}{E_{t}\left[\mathcal{E}_{\eta}^{1}\left(M_{t+1}\right)\right]}=1$.

If the agents that exhibit doubt (resp. overconfidence) have a risk tolerance higher (resp. lower) than the average, then the market price of risk in the heterogeneous beliefs setting is greater than in the standard setting.

Pessimism and doubt can then be seen as possible explanations for the riskpremium puzzle as well as for the risk-free rate puzzle as underlined by Abel (1989). However, it is not necessary to assume pessimism or doubt at the individual level nor on (equally weighted) average. A potential important source of pessimism or doubt at the aggregate level is the correlation between the individual risk tolerance and the individual level of pessimism/doubt.

\section{Conclusion}

In this paper, we provided an aggregation procedure which permits one to rewrite in a simple way the equilibrium characteristics (state price density, market price of risk, risk premium, risk-free rate) in a heterogeneous beliefs framework and to compare them with an otherwise similar standard setting. This procedure permits one to analyze in detail the impact of beliefs heterogeneity on the equilibrium characteristics.

In particular, we introduced concepts of pessimism and doubt and we proved, in a fairly general setting, that pessimism and doubt at the aggregate level lead to an increase of the market price of risk and to a decrease of the risk free rate. We also have shown how pessimism and doubt are transmitted from the individual to the aggregate level.

It appears that pessimism and doubt lead to an increase of the market price of risk and, under some additional conditions on the cautiousness level, to a decrease of the risk free rate. Furthermore, for utility functions in the HARA class, the 
aggregate level of pessimism and/or doubt is a weighted average of the individual levels of pessimism and doubt. These weights are proportional to the individual risk tolerances and, at the equilibrium, there is a bias toward the beliefs of the more risk tolerant agents. The intuition leads us to think that risk tolerant agents are more optimistic. It remains to prove that this intuition is correct and this could be done through behavioral experimental studies or through the introduction of a theoretical model of learning and beliefs construction. This is left for future research. 


\section{Appendix}

Proof of Proposition 2.1 Since $q^{*}$ is an interior equilibrium price process relative to the beliefs $\left(M^{i}\right)$, and the income processes $e^{i}$, we know that $\sum_{i=1}^{N} y^{*^{i}}=$ $e^{*}$ and that there exist positive Lagrange multipliers $\left(\lambda_{i}\right)$ such that for all $i$ and for all $t$,

$$
M_{t}^{i} u_{i}^{\prime}\left(t, y_{t}^{*^{i}}\right)=\lambda_{i} q_{t}^{*}
$$

We consider the maximization problem

$$
\left(\mathcal{P}^{\lambda}\right): \max \sum_{i=1}^{N} \frac{1}{\lambda_{i}} U_{i}\left(y^{i}\right) \text { under the constraint } \sum_{i=1}^{N} y^{i} \leq e^{*},
$$

where $U_{i}(c)=E\left[\sum_{t=0}^{T} u_{i}\left(t, c_{t}\right)\right]$. Denoting the solution by $\left(y^{i, \lambda}\right)_{i}$, we get that $\sum_{i=1}^{N} y^{i,(\lambda)}=e^{*}$ and the process $\left(\frac{1}{\lambda_{i}} u_{i}^{\prime}\left(t, y_{t}^{i,(\lambda)}\right)\right)_{t}$ is independent from $i$. We denote this process by $p^{(\lambda)}$. Letting $M^{(\lambda)} \equiv \frac{q^{*}}{p^{(\lambda)}}$, we then have for all $i$ and for all $t$

$$
M_{t}^{(\lambda)} u_{i}^{\prime}\left(t, y_{t}^{i,(\lambda)}\right)=M_{t}^{i} u_{i}^{\prime}\left(t, y_{t}^{*^{i}}\right) .
$$

The process $M^{(\lambda)}$ is adapted and positive. Moreover, at date $t=0$, we have for all $i, M_{0}^{i}=1$, and $\sum_{i=1}^{N y_{i},(\lambda)} i=\sum_{i=1}^{N} y_{0}^{*^{i}}=e_{0}^{*}$, so that $M_{0}^{(\lambda)}=1$. Then, it suffices to take $M=M^{(\lambda)}$ and $\bar{y}^{i}=y^{i,(\lambda)}$.

As far as uniqueness is concerned, notice that any process $y^{i}$ such that $\sum_{i=1}^{N} y^{i}=$ $e^{*}$ and

$$
M_{t} u_{i}^{\prime}\left(t, y_{t}^{i}\right)=M_{t}^{i} u_{i}^{\prime}\left(t, y_{t}^{*^{i}}\right)
$$

for some positive process $M$ is a solution of the maximization problem $\left(\mathcal{P}^{(\lambda)}\right)$. The uniqueness follows from the strict concavity of this maximization program.

Proof of Proposition 2.2 Similar to the proof of the analogous result in a standard setting.

Proof of Example 2.3 Since the representative utility function $u$ is given by

$$
u_{\lambda}(t, x)=\max _{\sum_{i=1}^{N} x_{i} \leq x} \sum_{i=1}^{N} \frac{1}{\lambda_{i}} u_{i}\left(t, x_{i}\right)
$$

the expression of $u_{\lambda}$ in the specific setting of linear risk tolerance utility functions is obtained as in the standard case (see e.g. Huang-Litzenberger, 1988). 

as

The expression of $M$ is obtained by using $M_{t} u_{i}^{\prime}\left(t, \bar{y}_{t}^{i}\right)=M_{t}^{i} u_{i}^{\prime}\left(t, y_{t}^{*^{i}}\right)$, as well

$$
\sum_{i=1}^{N} y^{*^{i}}=\sum_{i=1}^{N} \bar{y}^{i}=e^{*}
$$

Indeed, in the case of exponential utility functions, we have for all $i$,

$$
M^{i} \exp \left(-\frac{y^{*^{i}}}{\theta_{i}}\right)=M \exp \left(-\frac{\bar{y}^{i}}{\theta_{i}}\right)
$$

hence

$$
\prod_{i=1}^{N}\left(M^{i}\right)^{\theta_{i}} \exp \left(-\sum_{i=1}^{N} y^{*^{i}}\right)=M^{\bar{\theta}} \exp \left(-\sum_{i=1}^{N} \bar{y}^{i}\right),
$$

or equivalently

$$
M=\prod_{i=1}^{N}\left(M^{i}\right)^{\frac{\theta_{i}}{\bar{\theta}}} .
$$

In the case of power utility functions, we get for all $i$,

$$
M^{i}\left(\theta_{i}+\eta y^{*^{i}}\right)^{-1 / \eta}=M\left(\theta_{i}+\eta \bar{y}^{i}\right)^{-1 / \eta}=\lambda_{i} M\left(\bar{\theta}+\eta e^{*}\right)^{-1 / \eta} b
$$

hence

$$
\left(M^{i}\right)^{\eta} \lambda_{i}^{-\eta}=M^{\eta}\left(\bar{\theta}+\eta e^{*}\right)^{-1} b^{\eta}\left(\theta_{i}+\eta y^{*^{i}}\right)
$$

and

$$
M=\left[\sum_{i=1}^{N} \frac{\lambda_{i}^{-\eta}}{\sum_{i=1}^{N} \lambda_{i}^{-\eta}}\left(M^{i}\right)^{\eta}\right]^{1 / \eta}
$$

Proof of Proposition 2.4 Immediate defining $B$ and $\bar{M}$ by

$$
B_{t}=B_{t-1} \frac{E_{t-1}\left[M_{t}\right]}{M_{t-1}}, \quad B_{0}=1
$$

and

$$
\bar{M}_{t}=\frac{M_{t}}{B_{t}}
$$


Proof of Example 2.5 By Example (2.3) and Proposition (2.4), we immediately get the expressions for $B_{t}$. Hölder 's inequality permits us to conclude in the exponential case. In the case of power utility functions, for $0<\eta<1$, we get by Minkowski's inequality that $E_{t-1}\left[\left[\sum_{i=1}^{N} \gamma_{i}\left(M_{t}^{i}\right)^{\eta}\right]^{1 / \eta}\right] \leq\left[\sum_{i=1}^{N} \gamma_{i}\left(M_{t-1}^{i}\right)^{\eta}\right]^{1 / \eta}$ so that $B$ is nonincreasing. The case $\eta>1$ can be treated similarly.

Proof of the CCAPM formula and the risk free rate expression

We know that for any asset with associated price process $\left(S_{t}\right)_{t=0}^{T}$, we must have

$$
q_{t}^{*} S_{t}=E_{t}^{P}\left[q_{t+1}^{*} S_{t+1}\right] \text { for all } t=0, \ldots, T-1 .
$$

or equivalently, since $\widehat{M}_{t} B_{t} u^{\prime}\left(t, e_{t}^{*}\right)=q_{t}^{*}$,

$$
S_{t}=E_{t}^{p}\left[\frac{\widehat{M}_{t+1} B_{t+1} u^{\prime}\left(t+1, e_{t+1}^{*}\right)}{\widehat{M}_{t} B_{t} u^{\prime}\left(t, e_{t}^{*}\right)} S_{t+1}\right]
$$

or by definition of $R_{t+1}$

$$
1=E_{t}^{p}\left[\frac{\widehat{M}_{t+1} B_{t+1} u^{\prime}\left(t+1, e_{t+1}^{*}\right)}{\widehat{M}_{t} B_{t} u^{\prime}\left(t, e_{t}^{*}\right)}\left(1+R_{t+1}\right)\right] .
$$

This leads to

$$
1=E_{t}^{p}\left[\frac{\widehat{M}_{t+1} B_{t+1} u^{\prime}\left(t+1, e_{t+1}^{*}\right)}{\widehat{M}_{t} B_{t} u^{\prime}\left(t, e_{t}^{*}\right)}\right]\left(1+E_{t}^{P}\left[R_{t+1}\right]\right)+\operatorname{cov}_{t}^{P}\left(\frac{\widehat{M}_{t+1} B_{t+1} u^{\prime}\left(t+1, e_{t+1}^{*}\right)}{\widehat{M}_{t} B_{t} u^{\prime}\left(t, e_{t}^{*}\right)}, R_{t+1}\right) .
$$

Now, applying equation (5.1) to the riskless asset yields $E_{t}^{p}\left[\frac{\widehat{M}_{t+1} B_{t+1} u^{\prime}\left(t+1, e_{t+1}^{*}\right)}{\widehat{M}_{t} B_{t} u^{\prime}\left(t, e_{t}^{*}\right)}\right]=$ $\frac{1}{1+r_{t+1}^{f}}$, hence

$$
1+r_{t+1}^{f}=\left(1+E_{t}^{P}\left[R_{t+1}\right]\right)+\left(1+r_{t+1}^{f}\right) \operatorname{cov}_{t}^{P}\left(\frac{\widehat{M}_{t+1} B_{t+1} u^{\prime}\left(t+1, e_{t+1}^{*}\right)}{\widehat{M}_{t} B_{t} u^{\prime}\left(t, e_{t}^{*}\right)}, R_{t+1}\right)
$$

and

$$
E_{t}^{P}\left[R_{t+1}\right]-r_{t+1}^{f}=-\frac{\operatorname{cov}_{t}^{P}\left(\frac{\widehat{M}_{t+1} B_{t+1} u^{\prime}\left(t+1, e_{t+1}^{*}\right)}{\widehat{M}_{t} B_{t} u^{\prime}\left(t, e_{t}^{*}\right)}, R_{t+1}\right)}{E_{t}^{p}\left[\frac{\widehat{M}_{t+1} B_{t+1} u^{\prime}\left(t+1, e_{t+1}^{*}\right)}{\widehat{M}_{t} B_{t} u^{\prime}\left(t, e_{t}^{*}\right)}\right]}
$$


or equivalently

$$
\begin{aligned}
E_{t}^{P}\left[R_{t+1}\right]-r_{t+1}^{f} & =-\operatorname{cov}_{t}^{P}\left[\frac{\bar{M}_{t+1} B_{t+1} u^{\prime}\left(t+1, e_{t+1}^{*}\right)}{E_{t}^{P}\left[\bar{M}_{t+1} B_{t+1} u^{\prime}\left(t+1, e_{t+1}^{*}\right)\right]}, R_{t+1}\right] \\
& =-\operatorname{cov}_{t}^{P}\left[\frac{\widehat{M}_{t+1} u^{\prime}\left(t+1, e_{t+1}^{*}\right)}{E_{t}^{P}\left[\widehat{M}_{t+1} u^{\prime}\left(t+1, e_{t+1}^{*}\right)\right]}, R_{t+1}\right]
\end{aligned}
$$

Proof of Proposition 3.3 1. Consider first the case of a pessimistic probability measure $\widehat{P}$. We have seen (Inequality 3.2 ) that the probability measures leading to an increase of the market price of risk are characterized by $\operatorname{cov}_{t}^{P_{u}}\left(\widehat{M}_{t+1}, e_{t+1}^{*}\right) \leq 0$ where $\frac{d P_{u}}{d P}$ is given (up to a constant) by $u^{\prime}\left(t+1, e_{t+1}^{*}\right)$. By definition, since $\widehat{P}$ is pessimistic with respect to $e^{*}$, the random variables $\widehat{M}_{t+1}$ and $-e_{t+1}^{*}$ are comonotonic conditionally to $F_{t}$. For positive random variables $X$ and $Z$ such that $X$ and $Z$ are comonotonic conditionally to $F_{t}$, we have $\operatorname{cov}_{t}^{Q}(X, Z) \geq 0$ for any probability measure $Q$ absolutely continuous with respect to $P$. Indeed, we have

$$
\begin{aligned}
\operatorname{cov}_{t}^{Q}(X, Z) & =E_{t}^{Q}[X Z](\omega)-E_{t}^{Q}[X](\omega) E_{t}^{Q}[Z](\omega) \\
& =\int\left(x-x^{\prime}\right)\left(y-y^{\prime}\right) M(\omega, d(x, y)) \otimes M\left(\omega, d\left(x^{\prime}, y^{\prime}\right)\right)
\end{aligned}
$$

where $M(\omega,$.$) is the conditional law of (x, y)$ with respect to $F_{t}$. It is clear then that $\operatorname{cov}_{t}^{Q}(X, Z) \geq 0$ and we easily deduce from there that $\operatorname{cov}_{t}^{P_{u}}\left(\widehat{M}_{t+1}, e_{t+1}^{*}\right) \leq 0$.

2. Consider now the case of a probability measure $\widehat{P}$ which exhibits doubt. We have obtained (Inequality 3.1 ) that $\widehat{P}$ leads to a higher market price of risk if and only if

$$
\frac{E_{t}^{\widehat{P}}\left[u^{\prime}\left(e_{t+1}^{*}\right) e_{t+1}^{*}\right]}{E_{t}^{\widehat{P}}\left[u^{\prime}\left(e_{t+1}^{*}\right)\right]} \leq \frac{E_{t}^{P}\left[u^{\prime}\left(e_{t+1}^{*}\right) e_{t+1}^{*}\right]}{E_{t}^{P}\left[u^{\prime}\left(e_{t+1}^{*}\right)\right]} .
$$

We have

$$
\frac{E_{t}^{\widehat{P}}\left(e_{t+1}^{*} u^{\prime}\left(e_{t+1}^{*}\right)\right)}{E_{t}^{\widehat{P}}\left(u^{\prime}\left(e_{t+1}^{*}\right)\right)}=\frac{E_{t}^{P}\left[\left(e_{t+1}^{*}-m_{t}\right) \widehat{M}_{t+1} u^{\prime}\left(e_{t+1}^{*}\right)\right]}{E_{t}^{P}\left[\widehat{M}_{t+1} u^{\prime}\left(e_{t+1}^{*}\right)\right]}+m_{t}
$$


where $m_{t}=E_{t}\left[e_{t+1}^{*}\right]$. Since the random vector $\left(e_{t+1}^{*}-m_{t}, \widehat{M}_{t+1}\right)$ is distributed like $\left(m_{t}-e_{t+1}^{*}, \widehat{M}_{t+1}\right)$ conditionally to $F_{t}$, we have

$E_{t}^{P}\left[\left(e_{t+1}^{*}-m_{t}\right) \widehat{M}_{t+1} u^{\prime}\left(e_{t+1}^{*}\right) 1_{e_{t+1}^{*} \leq m_{t}}\right]=E_{t}^{P}\left[\left(m_{t}-e_{t+1}^{*}\right) \widehat{M}_{t+1} u^{\prime}\left(2 m_{t}-e_{t+1}^{*}\right) 1_{e_{t+1}^{*} \geq m_{t}}\right]$

hence

$E_{t}^{P}\left[\left(e_{t+1}^{*}-m_{t}\right) \widehat{M}_{t+1} u^{\prime}\left(e_{t+1}^{*}\right)\right]=E_{t}^{P}\left[\left(m_{t}-e_{t+1}^{*}\right) \widehat{M}_{t+1}\left[u^{\prime}\left(2 m_{t}-e_{t+1}^{*}\right)-u^{\prime}\left(e_{t+1}^{*}\right)\right] 1_{e_{t+1}^{*} \geq m_{t}}\right]$.

Then

$\frac{E_{t}^{\widehat{P}}\left(e_{t+1}^{*} u^{\prime}\left(e_{t+1}^{*}\right)\right)}{E_{t}^{\widehat{P}}\left(u^{\prime}\left(e_{t+1}^{*}\right)\right)}=\frac{E_{t}^{P}\left[\left(m_{t}-e_{t+1}^{*}\right) \widehat{M}_{t+1}\left[u^{\prime}\left(2 m_{t}-e_{t+1}^{*}\right)-u^{\prime}\left(e_{t+1}^{*}\right)\right] 1_{e_{t+1}^{*} \geq m_{t}}\right]}{E_{t}^{P}\left[\widehat{M}_{t+1}\left[u^{\prime}\left(2 m_{t}-e_{t+1}^{*}\right)+u^{\prime}\left(e_{t+1}^{*}\right)\right] 1_{e_{t+1}^{*} \geq m_{t}}\right]}+m_{t}$.

We want to compare this quantity with

$$
\frac{E_{t}^{P}\left[\left(m_{t}-e_{t+1}^{*}\right)\left[u^{\prime}\left(2 m_{t}-e_{t+1}^{*}\right)-u^{\prime}\left(e_{t+1}^{*}\right)\right] 1_{e_{t+1}^{*} \geq m_{t}}\right]}{E_{t}^{P}\left[\left[u^{\prime}\left(2 m_{t}-e_{t+1}^{*}\right)+u^{\prime}\left(e_{t+1}^{*}\right)\right] 1_{e_{t+1}^{*} \geq m_{t}}\right]}+m_{t} .
$$

Letting $g\left(m_{t}, e_{t+1}^{*}\right) \equiv\left(m_{t}-e_{t+1}^{*}\right)\left[u^{\prime}\left(2 m_{t}-e_{t+1}^{*}\right)-u^{\prime}\left(e_{t+1}^{*}\right)\right]$ and $h\left(m_{t}, e_{t+1}^{*}\right) \equiv$ $u^{\prime}\left(2 m_{t}-e_{t+1}^{*}\right)+u^{\prime}\left(e_{t+1}^{*}\right)$, we are led to compare $\frac{E_{t}^{P}\left[\widehat{M}_{t+1} g\left(m_{t}, e_{t+1}^{*}\right) 1_{e_{t+1}^{*} \geq m_{t}}\right]}{E_{t}^{P}\left[\widehat{M}_{t+1} h\left(m_{t}, e_{t+1}^{*}\right) 1_{e_{t+1}^{*} \geq m_{t}}\right]}$ with $\frac{E_{t}^{P}\left[g\left(m_{t}, e_{t+1}^{*}\right) 1_{e_{t+1}^{*} \geq m_{t}}\right]}{E_{t}^{P}\left[h\left(m_{t}, e_{t+1}^{*}\right) 1_{e_{t+1}^{*} \geq m_{t}}\right]}$. Let us now define the probability measure $P^{g}$ by $\frac{d P^{g}}{d P}=$ $\frac{g\left(m_{t}, e_{t+1}^{*}\right) 1_{e_{t+1}^{*} \geq m_{t}}}{E^{P}\left[g\left(m_{t}, e_{t+1}^{*}\right) 1_{e_{t+1}^{*} \geq m_{t}}^{*}\right]}$. We are led to compare $\frac{E_{t}^{P^{g}}\left[\widehat{M}_{t+1}\right]}{E_{t}^{P^{g}}\left[\widehat{M}_{t+1} \frac{h}{g}\left(m_{t}, e_{t+1}^{*}\right)\right]}$ with $\frac{1}{E_{t}^{P^{g}\left[\frac{h}{g}\left(m_{t}, e_{t+1}^{*}\right)\right]}}$.

It is easy to check that the function $\frac{h}{g}(x, \cdot): y \mapsto \frac{\left(u^{\prime}(x+y)+u^{\prime}(x-y)\right)}{y\left(u^{\prime}(x-y)-u^{\prime}(x+y)\right)}$ is decreasing on $\mathbb{R}_{+}$, so that $\widehat{M}_{t+1} 1_{e_{t+1}^{*} \geq m_{t}}$ and $-\frac{h}{g}\left(m_{t}, e_{t+1}^{*}\right) 1_{e_{t+1}^{*} \geq m_{t}}$ are comonotonic conditionally to $F_{t}$. We have then, as seen in 1 ., $\operatorname{cov}_{t}^{P^{g}}\left(\frac{h}{g}\left(m_{t}, e_{t+1}^{*}\right), \widehat{M}_{t+1}\right) \leq 0$ or equivalently

$$
E_{t}^{P^{g}}\left[\widehat{M}_{t+1} \frac{h}{g}\left(m_{t}, e_{t+1}^{*}\right)\right] \leq E_{t}^{P^{g}}\left[\widehat{M}_{t+1}\right] E_{t}^{P^{g}}\left[\frac{h}{g}\left(m_{t}, e_{t+1}^{*}\right)\right]
$$

which concludes the proof. 
Proof of Proposition 3.4 1. If $\widehat{P}$ exhibits pessimism with respect to $e^{*}$, then by definition of pessimism and since $u$ is concave, we easily obtain that for all $t, \bar{M}_{t+1}$ and $u^{\prime}\left(e_{t+1}^{*}\right)$ are comonotonic conditionally to $F_{t}$. As seen in the proof of the previous proposition, we deduce that for all probability measure $\widehat{P}$ absolutely continuous with respect to $P$, we have for all $t, \operatorname{cov}_{t}^{\widehat{P}}\left(\bar{M}_{t+1}, u^{\prime}\left(e_{t+1}^{*}\right)\right) \geq$ 0 . In particular, we have for all $t, \operatorname{cov}_{t}^{P}\left(\bar{M}_{t+1}, u^{\prime}\left(e_{t+1}^{*}\right)\right) \geq 0$ and $E_{t}^{\widehat{P}}\left[u^{\prime}\left(e_{t+1}^{*}\right)\right]$ $\geq E_{t}^{P}\left[u^{\prime}\left(e_{t+1}^{*}\right)\right]$.

2. If $\widehat{P}$ exhibits doubt with respect to $e^{*}$, then we know that

$$
E_{t}^{\widehat{P}}\left[u^{\prime}\left(e_{t+1}^{*}\right)\right]=E_{t}^{\widehat{P}}\left[\left[u^{\prime}\left(2 m_{t}-e_{t+1}^{*}\right)+u^{\prime}\left(e_{t+1}^{*}\right)\right] 1_{e_{t+1}^{*} \geq m_{t}}\right]
$$

and

$$
E_{t}^{P}\left[u^{\prime}\left(e_{t+1}^{*}\right)\right]=E_{t}^{P}\left[\left[u^{\prime}\left(2 m_{t}-e_{t+1}^{*}\right)+u^{\prime}\left(e_{t+1}^{*}\right)\right] 1_{e_{t+1}^{*} \geq m_{t}}\right] .
$$

Since $u$ has a convex derivative, $u^{\prime}(2 m-x)+u^{\prime}(x)$ is nondecreasing on $x \geq m$ and we conclude as in the previous case.

Proof of Proposition 3.5 1. Immediate consequence of Proposition 3.3 and the fact that the market price of risk under heterogeneous beliefs is the same as the market price of risk under the subjective belief $Q$.

2. Immediate consequence of Equality 2.6, Proposition 3.4 and Example 2.5.

Proof of Proposition 4.1 In the exponential utility case, we have $\frac{\mathcal{E}_{0}^{1}\left(M_{t+1}\right)}{E_{t}\left[\mathcal{E}_{0}^{1}\left(M_{t+1}\right)\right]}=$ 1 and

$$
\begin{aligned}
\mathcal{E}_{0}^{\theta \cdot}\left(M_{t+1}^{\cdot}\right) & =\prod_{i=1}^{N}\left(M_{t+1}^{i}\right)^{\left(\frac{\theta_{i}}{\theta}-\frac{1}{N}\right)} \mathcal{E}_{0}^{\mathbf{1}}\left(M_{t+1}^{\cdot}\right) \\
& =\prod_{i=1}^{N}\left(M_{t+1}^{i}\right)^{\left(\frac{\theta_{i}}{\theta}-\frac{1}{N}\right)} E_{t}\left[\mathcal{E}_{0}^{\mathbf{1}}\left(M_{t+1}^{\cdot}\right)\right]
\end{aligned}
$$

Consequently, $\bar{M}=\prod_{i=1}^{N}\left(M_{t+1}^{i}\right)^{\left(\frac{\theta_{i}}{\theta}-\frac{1}{N}\right)} N_{t}$ where $N_{t}$ is some $F_{t}$-measurable random variable. It is easy to see that under our assumptions, the random variable $M=\prod_{i=1}^{N}\left(M_{t+1}^{i}\right)^{\left(\theta_{i}-\frac{\overline{\bar{\theta}}}{N}\right) / \bar{\theta}}$ is comonotonic with $-e_{t+1}^{*}$ conditionally to $F_{t}$, hence the consensus probability is pessimistic. Proposition 3.3 concludes.

For power utility functions, let us remark that $\left[\mathcal{E}_{\eta}^{\gamma \cdot}\left(M_{t+1}\right)\right]^{\eta}=\sum_{i=1}^{N}\left(\gamma_{i}-\frac{1}{N}\right)\left(M_{t+1}^{i}\right)^{\eta}+$ $\left[\mathcal{E}_{\eta}^{\mathbf{1}}\left(M_{t+1}\right)\right]^{\eta}$. Under our assumptions, since $\left[\mathcal{E}_{\eta}^{\mathbf{1}}\left(M_{t+1}\right)\right]^{\eta}$ is $F_{t}$-measurable, this 
expression is comonotonic with $-e_{t+1}^{*}$ conditionally to $F_{t}$ and again, Proposition 3.3 concludes.

Proof of Proposition 4.2 For $\eta=0$, as in the proof of Proposition ??, we have $\bar{M}=\prod_{i=1}^{N}\left(M_{t+1}^{i}\right)^{\left(\frac{\theta_{i}}{\theta}-\frac{1}{N}\right)} N_{t}$ where $N_{t}$ is some $F_{t}$-measurable random variable. Now, if the more risk tolerant agents (i.e. those for which $\theta_{i}>\frac{\bar{\theta}}{N}$ ) exhibit doubt (i.e. have a density $M_{t+1}^{i}$ that is symmetric with respect to $E_{t}\left[e_{t+1}^{*}\right]$ nondecreasing after $E_{t}\left[e_{t+1}^{*}\right]$ conditionally to $F_{t}$ ) and if the less risk tolerant agents (i.e. those for which $\theta_{i}<\frac{\bar{\theta}}{N}$ ) are overconfident (i.e. have a density $M_{t+1}^{i}$ that is nonincreasing with $e_{t+1}^{*}$ after $E_{t}\left[e_{t+1}^{*}\right]$ conditionally to $F_{t}$ ), we clearly obtain that $\bar{M}$ exhibits doubt.

The case $\eta \neq 0$ can be similarly treated using the arguments of the proof of Propositions 4.1 .

\section{References}

[1] Abel, A., 1989. Asset prices under heterogeneous beliefs: implications for the equity premium. Rodney L. White Center for Financial Research Working Papers, 09-89.

[2] Abel, A., 2002. An exploration of the effects of pessimism and doubt on asset returns. Journal of Economic Dynamics and Control, 26, 1075-1092.

[3] Calvet, L., Grandmont, J.-M., and I. Lemaire, 2004. Aggregation of Heterogenous Beliefs and Asset Pricing in Complete Financial Markets. CREST Working Paper, 2004-12, http://www.crest.fr/doctravail/document/2004-12.pdf.

[4] Cecchetti, S., Lam, P. and N. Mark, 2000. Asset pricing with distorded beliefs: are equity returns too good to be true? American Economic Review, 90, 787805.

[5] Cragg, J. and B. Malkiel, 1982. Expectations and the Structure of Share Prices. University of Chicago Press, Chicago.

[6] Detemple, J. and S. Murthy, 1994. Intertemporal Asset Pricing with Heterogeneous Beliefs. Journal of Economic Theory, 62, 294-320.

[7] Gallmeyer, M., 2000. Beliefs and volatility. GSIA Working Papers, Carnegie Mellon University, Tepper School of Business, 2000-E42. 
[8] Gallmeyer, M. and B. Hollifield, 2002. An examination of heterogeneous beliefs with a short sale constraint. Working paper, Carnegie Mellon University, Tepper School of Business, 2002-E2, http://chinook.tepper.cmu.edu/constraint.pdf.

[9] Genotte, G., 1986. Optimal portfolio choice under incomplete information. Journal of Finance, 41, 733-746.

[10] Giordani, P. and P. Söderlind, 2003. Is there evidence of pessimism and doubt in subjective distribution? A comment on Abel. CEPR Discussion Paper 4068, http://www.cepr.org/pubs/new-dps/dplist.asp?dpno=4068.

[11] Gollier, C., 2003, "Who should we believe? Collective RiskTaking Decisions with Heterogeneous Beliefs". IDEI Discussion Paper, http://idei.fr/doc/wp/2003/go_dt_0703.pdf.

[12] Harris, M. and A. Raviv, 1993. Differences of opinion make a horse race, Review of Financial Studies, 6 (3), 473-506.

[13] Huang, C.-F., and R. Litzenberger, 1988. Foundations of Financial Economics, Prentice Hall, Englewood Cliffs, New-Jersey.

[14] Jouini, E., and C. Napp, 2004. Consensus consumer and intertemporal asset pricing with heterogeneous beliefs. CEREMADE Working Paper 2004-65, http://www.ceremade.dauphine.fr/preprints/.

[15] Jouini, E. and C. Napp, 2005. On Abel's concept of doubt and pessimism. CEREMADE Working Paper 2005-25, http://www.ceremade.dauphine.fr/preprints/.

[16] Kocherlakota, N. R., 1996. The Equity Premium: It's Still a Puzzle. Journal of Economic Literature, 34, 42-71.

[17] Mehra, R., and E. Prescott, 1985. The Equity Premium: A Puzzle. Journal of Monetary Economics, 15, 145-162.

[18] Varian, H., 1985. Divergence of Opinion in Complete Markets. Journal of Finance, 40, 309-317.

[19] Varian, H., 1989. Difference of Opinion in Financial Markets. In Financial Risk: Theory, Evidence, and Implications. Stone C.C. (Ed.), Kluwer, Dordrecht, The Netherlands. 
[20] Weil, P., 1989. The Equity Premium Puzzle and the Risk Free Rate Puzzle. Journal of Monetary Economics, 24, 401-421.

[21] Williams, J. T., 1977. Capital Asset Prices with Heterogeneous Beliefs. Journal of Financial Economics, 5, 219-239.

[22] Zapatero, F., 1998. Effects of Financial Innovations on Market volatility when Beliefs are Heterogeneous. Journal of Economics, Dynamics and Control, 22, 597-626. 